\title{
Incrementally non-linear plasticity applied to rock joint modelling
}

\author{
Jérôme Duriez, Félix Darve and Frédéric-Victor Donzé
}

\begin{abstract}
Rock joint constitutive modelling is discussed through two new rock joint constitutive relations and a discrete numerical model. Regarding the constitutive relations, we emphasise the number of "tensorial zones", i.e. domains of constitutive incremental linearity, they involve: four zones for the first (called "quadrilinear"), and an infinite number for the second one (called "incrementally nonlinear"). Using these formulations, a large class of loading paths can be considered. Hardening through shearing and relations between the normal and tangential directions of the joint (e.g., dilatancy), can be described. Their predictive abilities are checked. Plastic features are included even if the relations are defined outside of the elasto-plastic formalism. These relations obey hence the physical evidences as the plastic limit criterion and flow rule. The flow rule is nonassociated, and the corresponding link with the nonsymmetry of the constitutive matrix is examined. Comparisons between the two relations and the discrete numerical model, i.e. a direct numerical simulation which is fundamentally different, are also discussed within the context of infilled rock joints.
\end{abstract}

\section{Introduction}

Many rock slopes present a behaviour ruled by the rock joints' mechanical properties. Hence rock joint constitutive modelling is the subject of this paper. Even when not planar, the geometry of the considered rock joints is supposed to be smooth enough so that a tangent plane can be taken into account. Furthermore, only one tangent direction in the plane is considered, resulting in a bidimensionnal mechanical state. This mechanical state is then defined by two scalar stresses: the normal $\sigma$ (considered to be positive in compression) and the tangential $\tau$, and by two scalar relative displacements: the normal component $u$ (considered to be positive in compression) and the tangential $\gamma$. Those variables are linked by the constitutive relation of the joint. A direct formalism $(\sigma, \tau)=f(u, \gamma)$ should be avoided, as the same "deformation" state of the joint can lead to different stress states. During a compression of rock joint, for example, due to the observed inelasticity [1], two different values of $\sigma$ can be obtained for the same value of $u$, whether this $u$-value is reached 
in loading or unloading. Consequently, we will use an incremental formalism $\overrightarrow{d \sigma}=(d \sigma, d \tau)=f_{h}(\overrightarrow{d l})=f_{h}(d u, d \gamma)$, with the function $f_{h}$ depending on the state variables and the hardening parameter $h$. From the rock joint's rateindependent behaviour and Euler's identity for homogeneous functions, we obtain the existence of an elasto-plastic matrix $M_{h}$ that depends only on direction $\vec{d}$ of $\overrightarrow{d l}(\vec{d}=\overrightarrow{d l} /\|\overrightarrow{d l}\|)$ (see Duriez et al. [2] for more details):

$$
\left(\begin{array}{c}
d \tau \\
d \sigma
\end{array}\right)=M_{h}(\vec{d})\left(\begin{array}{c}
d \gamma \\
d u
\end{array}\right)
$$

Different levels of model complexity are reached depending on how the matrix $M_{h}$ evolves with $\vec{d}$. If $M_{h}$ is constant for any given $\vec{d}$, the relation is eventually incrementally linear and can describe only elastic behaviours, which is not comprehensive. Tensorial zones [3, 4] are then defined as sections of the $(d \gamma, d u)$ plane in which $M_{h}$ is constant and equal to one unique matrix. The last example of elasticity corresponds to a case with one tensorial zone and illustrates that a greater number of tensorial zones is required.

Usual rock joint constitutive relations are generally not consistent with this required incremental formalism, with a high number of tensorial zones. Previous works by Bandis et al. [1] and Barton et al. [5] have proposed relations directly linking stresses to relative displacements that do not obey the incremental formalism mentioned previously. Incrementally linear constitutive relations for rock joints have also been previously proposed $[6,7]$. Their limitation is that they present only one tensorial zone, and hence are unable to reproduce the inelastic behaviours of rock joints, as explained above. This inability can be corrected by elasto-plastic relations $[8,9,10]$. We also mention work by Souley et al. [11], which improves on the results of Saeb \& Amadei [7] from this point of view. Nevertheless, such relations still present only two tensorial zones, which is a limited number. Indeed, Hill's results $[12,13,14]$ suggest that a finite number of tensorial zones is adequate for metal crystals, but not for geomaterials. The number of tensorial zones required corresponds to the different types of plastic deformation mechanisms. In contrast to metals whose plasticity is ruled by the sliding of dislocations, geomaterials seem not to have such special mechanisms $[15,16]$. Simulating their behaviour thus requires the use of an infinite number of tensorial zones. The incrementally non-linear, second order, (INL2) relations initially developed by Darve for soils $[3,17]$ present this feature: the matrix $M_{h}$ depends on the direction $\vec{d}$ in a continuous manner. For a general discussion of incremental nonlinearity, see Darve \& Nicot [18]. Incremental nonlinearity has been previously adapted to rock joints modelling $[19,2]$. The same construction will be used here, leading to a new INL2 relation that improves on the previous work of Duriez et al. [2].

Section 2 then presents the construction of two rock joint constitutive relations: one INL2, and one quadrilinear (Quadri). The quadrilinear formalism corresponds to the octolinear relation (Octo) developed for soils in parallel with 
the INL2 relations. Even if the Octo formalism provides eight tensorial zones rather than an infinite number, its results are nevertheless comparable to those of INL2 [20] (or section 5.1 of this paper). Here, the Quadri relation presents only four tensorial zones, which is still higher than the usual rock joint relations, but it allows the same easy analytical analysis, as Octo. Comparisons with the INL2 formalism is one of the goals of this paper, showing that the results are generally close, so that the analytical results obtained in Quadri case are usually valid in the INL2 one.

These relations are calibrated in section 3 . In that section, the relations are then validated by testing their predictive abilities. The calibration and validation of these relations rely on the numerical results presented by Duriez et al. [2]. These results were obtained from a rock joint numerical model using Discrete Element Methods, DEM, see Cundall \& Strack [21]. In [2], the discrete results were compared with experimental ones. Thus, no other experimental result will be presented. We note that these discrete results have already been used in [2] to define an INL2 relation. The one presented here has some features in common with this previous relation, but significant improvements have been made. We also note that the numerical model of [2] considered infilled rock joints. The framework of this previous work was indeed rock slope stability analysis, and in this case such rock joints are critical, due to their small shear strength $[22,23,24]$. This assumption of a special type of rock joint influences the values of the parameters which will be defined but not the way developments will be carried out. We mention here some general considerations about constitutive relations $\overrightarrow{d \sigma}=f(\overrightarrow{d l})$, which apply to any type of rock joint, including the perhaps more general case of rough surfaces contact.

The final sections discuss the proposed relations in the plasticity framework and examine their theoretical basis. The plastic limit criterion is considered in section 4, the response envelopes in section 5, and the flow rule in section 6 . Emphasis will again be placed on comparing the Quadri and INL2 formalisms and also with the discrete numerical results of the infilled rock joint model.

\section{General definition of two rock joint constitu- tive relations}

Section 2.1 presents the construction of the two rock joint constitutive relations, that are established outside the usual elasto-plasticity framework. In this sense, no plasticity limit criterion, for example, is clearly stated. Nevertheless, to be realistic, the proposed constitutive relations have to describe the stress states that are limited by such plasticity limit criterion. This factor will be discussed in detail in section 4 , but the fundamentals of the approach are presented in section 2.2 . 


\subsection{Construction of the relations}

The construction of a rock joint INL2 relation can be found in [2]. It leads to the following equation that links the (incremental) stresses existing along the joint to the (incremental) relative displacements:

$$
\left(\begin{array}{c}
d \tau \\
d \sigma
\end{array}\right)=\frac{1}{2}\left(P^{+}+P^{-}\right)\left(\begin{array}{c}
d \gamma \\
d u
\end{array}\right)+\frac{1}{2 \sqrt{d u^{2}+d \gamma^{2}}}\left(P^{+}-P^{-}\right)\left(\begin{array}{c}
d \gamma^{2} \\
d u^{2}
\end{array}\right)
$$

We recall, following [2], that the matrices $P^{+}$and $P^{-}$are defined through eight moduli:

$$
P^{+}=\left(\begin{array}{cc}
G_{\gamma}^{+} & G_{u}^{+} \\
N_{\gamma}^{+} & N_{u}^{+}
\end{array}\right) \quad P^{-}=\left(\begin{array}{cc}
G_{\gamma}^{-} & G_{u}^{-} \\
N_{\gamma}^{-} & N_{u}^{-}
\end{array}\right)
$$

These moduli, $G_{\gamma}^{+/-}, N_{\gamma}^{+/-}, G_{u}^{+/-}$and $N_{u}^{+/-}$, characterise the behaviour of the joints along two calibration paths:

- the first calibration path is a constant normal displacement (CND) shearing path that is controlled by $(d u=0 ; d \gamma=c s t)$. From the resulting changes in $\sigma$ and $\tau$, four moduli are defined:

$$
\begin{array}{rlrl}
G_{\gamma}^{+} & =\frac{\partial \tau}{\partial \gamma}{ }_{u, d \gamma>0} & G_{\gamma}^{-} & =\frac{\partial \tau}{\partial \gamma} \\
N_{\gamma}^{+}=\frac{\partial \sigma}{\partial \gamma} & N_{\gamma, d \gamma>0}^{-} & =\frac{\partial \sigma}{\partial \gamma}_{u, d \gamma<0}
\end{array}
$$

In these definitions, $d \gamma>0$ corresponds to the shear loading, while the unloading is associated with $d \gamma<0$. The $G_{\gamma}^{+/-}$moduli are similar to classical tangential rigidities. And the $N_{\gamma}^{+/-}$moduli describe the dilatant feature of the joint. Figure 1 illustrates the definitions of Equation (4).

- a second calibration path is the constant tangential displacement (CTD)
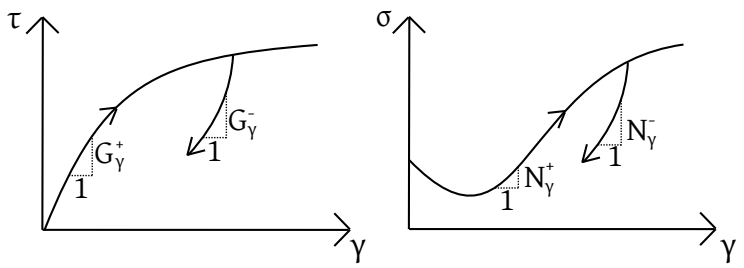

Figure 1: Definition of four moduli from an arbitrary CND path behaviour

path which is controlled by $(d u=c s t ; d \gamma=0)$. In this case, four other moduli can be defined:

$$
\begin{array}{rlrl}
G_{u}^{+} & =\frac{\partial \tau}{\partial u}{ }_{\gamma, d u>0} & G_{u}^{-} & =\frac{\partial \tau}{\partial u}_{\gamma, d u<0} \\
N_{u}^{+}=\frac{\partial \sigma}{\partial u}{ }_{\gamma, d u>0} & N_{u}^{-} & =\frac{\partial \sigma}{\partial u} \\
& &
\end{array}
$$


The $G_{u}^{+/-}$moduli describe how the tangential stress $\tau$ evolves during changes in the normal displacement $u$. Indeed Duriez et al. [2] discusses how compressions can affect the values of $\tau$ in cases where the joint has been previously sheared. The $N_{u}^{+/-}$moduli correspond to the classical normal rigidities. Figure 2 illustrates Equation (5).

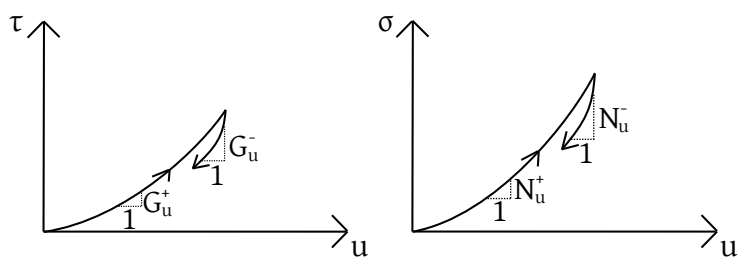

Figure 2: Definition of four other moduli from an arbitrary CTD path behaviour

We recall that Equation (2) corresponds to Equation (1), with continuous changes in $M_{h}$ with respect to $\vec{d}$. It also corresponds to an interpolation. In this case, the behaviour of the joint along any loading path is determined by the behaviours corresponding to the calibration paths through a nonlinear (quadratic) interpolation (see [25], for example). The other constitutive relation, Quadri, is defined if a piecewise linear interpolation is used. It comes with the matrices $P^{+}$and $P^{-}$in this case:

$$
\left(\begin{array}{c}
d \tau \\
d \sigma
\end{array}\right)=\frac{1}{2}\left(P^{+}+P^{-}\right)\left(\begin{array}{c}
d \gamma \\
d u
\end{array}\right)+\frac{1}{2}\left(P^{+}-P^{-}\right)\left(\begin{array}{l}
|d \gamma| \\
|d u|
\end{array}\right)
$$

To illustrate equations (2) and (6), we emphasise that, for the $(d u=0 ; d \gamma \neq 0)$ loading, we obtain:

$$
\begin{aligned}
& \left\{\begin{array}{c}
d \tau=\left(G_{\gamma}^{+}+G_{\gamma}^{-}\right) \frac{d \gamma}{2}+\left(G_{\gamma}^{+}-G_{\gamma}^{-}\right) \frac{d \gamma}{2 \sqrt{d \gamma^{2}}} \text { for INL2 case, Equation (2) } \\
\text { or } d \tau=\left(G_{\gamma}^{+}+G_{\gamma}^{-}\right) \frac{d \gamma}{2}+\left(G_{\gamma}^{+}-G_{\gamma}^{-}\right) \frac{|d \gamma|}{2} \text { for Quadri case, Equation (6) }
\end{array}\right. \\
& \Leftrightarrow d \tau=G_{\gamma}^{+} d \gamma \text { if } d \gamma>0 \text { and } d \tau=G_{\gamma}^{-} d \gamma \text { if } d \gamma<0, \text { for both cases }
\end{aligned}
$$

Equation (7) obviously corresponds to the definitions of the moduli, but it also expresses a classical elasto-plastic behaviour, with different loading and unloading stiffness.

The calibrations of the two constitutive relations (2) and (6) are identical: thus the responses of these two relations for calibration paths are identical. For other loading paths, both predictions are close, with the INL2 formalism being generally more precise (comparisons will be presented in this paper). However, the Quadri case has the advantage of making analytical computations possible. If constant signs for $d \gamma$ and $d u$ are assumed, which means to consider a given tensorial zone, then the Quadri relation is indeed linear. Expressions for the corresponding matrices $M_{h}$ are presented in Table 1 . As we will see, the analytical results obtained in this way can be extended to the INL2 case. 


\begin{tabular}{c|c|c}
$M_{h}$ & $d \gamma<0$ & $d \gamma>0$ \\
\hline$d u>0$ & $\left(\begin{array}{cc}G_{\gamma}^{-} & G_{u}^{+} \\
N_{\gamma}^{-} & N_{u}^{+}\end{array}\right)=M^{-+}$ & $\left(\begin{array}{cc}G_{\gamma}^{+} & G_{u}^{+} \\
N_{\gamma}^{+} & N_{u}^{+}\end{array}\right)=M^{++}$ \\
$d u<0$ & $\left(\begin{array}{cc}G_{\gamma}^{-} & G_{u}^{-} \\
N_{\gamma}^{-} & N_{u}-\end{array}\right)=M^{--}$ & $\left(\begin{array}{cc}G_{\gamma}^{+} & G_{u}^{-} \\
N_{\gamma}^{+} & N_{u}^{-}\end{array}\right)=M^{+-}$
\end{tabular}

Table 1: Constitutive matrices for the different tensorial zones of Quadri relation

\subsection{Link with a plasticity limit criterion}

As stated in the introduction, the use of equations (2) or (6) do not require the use of a plasticity limit criterion. However, such criteria are physical evidence and have to be fulfilled by any constitutive relation. For rock joints, it is always observed, for example, that the tangential stress $\tau$ reaches a peak and/or a plateau at the end of a constant normal load (CNL, with $\sigma=c s t$ ) shearing $[1,7,26,24,27]$. The consequences for the definition of constitutive relations are presented here.

Let us consider any incrementally linear rock joint constitutive relation, as defined by following Equation (8):

$$
\left(\begin{array}{c}
d \tau \\
d \sigma
\end{array}\right)=\left(\begin{array}{ll}
G_{\gamma} & G_{u} \\
N_{\gamma} & N_{u}
\end{array}\right)\left(\begin{array}{c}
d \gamma \\
d u
\end{array}\right)
$$

This relation holds for our Quadri relation in a given tensorial zone. In this framework, peaks or plateaus in $\tau$ for the CNL tests can be expressed mathematically. As the CNL conditions already imply that $d \sigma=0$, obtaining $d \tau=0$ at $\tau$ peak or $\tau$ plateau implies that:

$$
\begin{gathered}
\overrightarrow{d \sigma}=M \overrightarrow{d l}=\overrightarrow{0}, \text { with } \overrightarrow{d l} \neq \overrightarrow{0} \Leftrightarrow \operatorname{det}(M)=0 \\
\Leftrightarrow G_{\gamma} N_{u}-G_{u} N_{\gamma}=0 \Leftrightarrow G_{\gamma} N_{u}=G_{u} N_{\gamma}
\end{gathered}
$$

Let us note that $\operatorname{det}(M)=0$ corresponds exactly to the general definition of a plastic limit criterion in elasto-plastic theory.

The previous work by Duriez et al. [2] was incomplete in this aspect. A Mohr-Coulomb plasticity limit criterion was considered to limit the stress states: $\tau \leq \sigma \tan (\varphi)$. But the moduli expressions were not consistent with this plasticity limit criterion regarding Equation (9), as Figure 3 illustrates. The evolution of the determinants of the two matrices corresponding to $d \gamma>0$, taken as examples among four, are plotted according to $\tau$ from $\tau=0$ to $\tau=\tau_{\max }=\sigma \tan (\varphi)$. For each determinant of the two matrices, different constant values of $\sigma(1 ; 2$ and $5 \mathrm{MPa}$ ) are considered. It thus illustrates that, with moduli such as those defined in [2], the determinant of the matrices can cancel both on the plasticity limit criterion (maximum value of $\tau$ in the figure) and before the plasticity limit criterion, as in the case of the matrix corresponding to $d \gamma>0, d u<0$ (the typical case for a CNL test during the dilatant phase). This result will be 


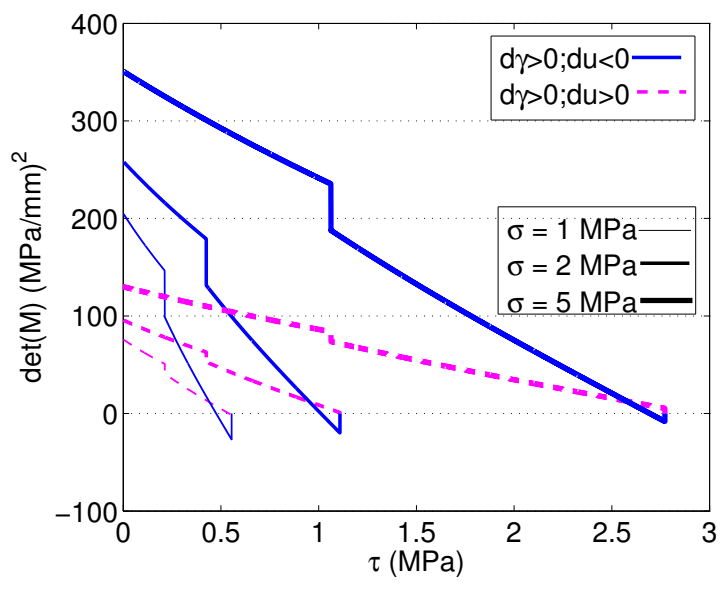

Figure 3: Determinant of two constitutive matrices of [2], according to $\tau$ (from nonsheared to completely sheared states), under different values of $\sigma$

improved in the present work.

To conclude this section, we note that Equation (9) can be physically interpreted. We can consider $N_{u}$ and $G_{\gamma}$ as diagonal rigidities in the sense that they link together the normal variables $(d \sigma$ with $d u)$ or the tangential variables $(d \tau$ with $d \gamma$ ), and hence $G_{u}$ and $N_{\gamma}$ as nondiagonal ones. Equation (9) thus states that on the plasticity limit criterion, the (product of) nondiagonal rigidities are as great as the (product of) diagonal ones. More generally, a link between these diagonal and nondiagonal terms is suggested through this equation. This result will be used in following calibration of our relations (Quadri and INL2). Such links also have the advantage of reducing the number of parameters.

\section{Calibration and validation of the relations}

Calibration and validation of the INL2 relation, not of the Quadri relation, have been proposed in [2]. As explained in section 1, past work has focused on granular infilled rock joints. Thus, a numerical model using DEM [21] (with the Yade code [28]) has been used to obtain a sufficient database for our calibration paths (CND and CTD). The choice of DEM was motivated by the presence of the granular filler, as many works show the quantitative and qualitative abilities of DEM to simulate granular media [29, 30, 31, 32, 33]. In [2], the DEM results were compared with some new experimental results: such comparison will therefore not be presented again.

In the present paper, the same DEM results will be used. However, for the reasons discussed previously in 2.2, a new calibration will be performed in 3.1. INL2 or Quadri relations present no differences in the calibration step. For 
validation, section 3.2 will examine the predictive abilities of these two relations and illustrate their differences.

\subsection{Calibration}

The Figure 4 shows the behaviour of the joint along CND paths, as determined by the DEM simulations [2] taken as reference. Different CND paths were performed, with different initial values for $\sigma_{0}$, in loading and unloading. Equation

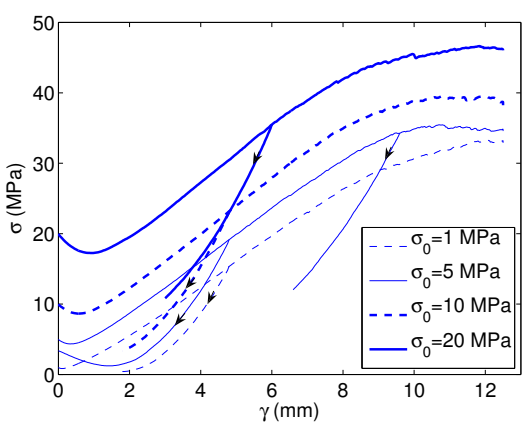

(a) $\sigma(\gamma)$

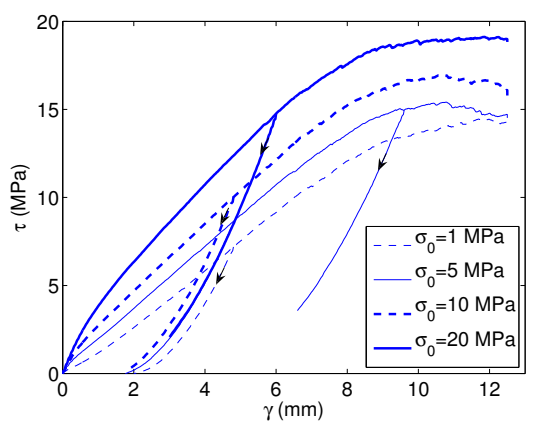

(b) $\tau(\gamma)$

Figure 4: Reference CND paths behaviour, from [2], obtained by DEM computations

(10) proposes new definitions of the corresponding moduli $\left(G_{\gamma}^{+/-}, N_{\gamma}^{+/-}\right)$, so that the constitutive relations provide the same response.

$$
\begin{aligned}
& N_{\gamma}^{+}=\left\{\begin{array}{cc}
N_{\gamma}^{0}<0 & \text { if } \tau / \sigma<\tan \left(\varphi_{c}\right) \\
N_{\gamma}^{+}{ }_{\text {dil }}>0 & \text { otherwise }
\end{array}\right. \\
& G_{\gamma}^{+}=G_{\gamma}^{0}-\left(G_{\gamma}^{0}-G_{\gamma}^{f}\right) \frac{1-e^{-\frac{\tau / \sigma}{\tan (\varphi)}}}{1-e^{-1}} \text { where } G_{\gamma}^{f}=N_{\gamma}^{+}{ }_{d i l} \tan (\varphi) \\
& N_{\gamma}^{-}=\left\{\begin{array}{cc}
N_{\gamma}^{0}<0 & \text { if } \tau / \sigma<\tan \left(\varphi_{c}\right) \\
\frac{G_{\gamma}^{0}}{\tan (\varphi)}>0 & \text { otherwise }
\end{array}\right. \\
& G_{\gamma}^{-}=G_{\gamma}^{0}
\end{aligned}
$$

The $N_{\gamma}^{+}$moduli takes first negative and then positive values, to describe the observed contractant-dilatant transition, as shown in Figure 4(a). The $G_{\gamma}^{+}$ moduli evolves continuously during shearing from an initial value of $G_{\gamma}^{0}$ to a final value of $G_{\gamma}^{f}$, as suggested by Figure 4(b). As discussed in [2], the shearing is taken into account through the ratio of stresses $\tau / \sigma$ (rather than the 
displacement $\gamma$, for example). The final value, $G_{\gamma}^{f}$, is expressed according to a parameter related to $N_{\gamma}^{+}, N_{\gamma}^{+}$dil (due to the discussion of the plastic limit criterion of section 2.2) and an other parameter, the angle $\varphi$.

The $N_{\gamma}^{-}$unloading moduli is expressed equal to the $N_{\gamma}^{+}$value in the contractant domain; otherwise, a link with $G_{\gamma}$ (and $\varphi$ ) is still used. Finally the $G_{\gamma}^{-}$unloading moduli is considered to be equal to the initial value of the corresponding loading moduli $G_{\gamma}^{+}$.

Figure 5 compares the DEM results with the INL2 or Quadri results, with such expressions of moduli. The stress levels observed in the DEM results are not reproduced with the constitutive relations. Such levels are considered to be artefacts caused by a numerical sample being no longer homogeneous for high values of $\gamma$. This issue will be discussed more carefully in section 6 . With the exception of these levels, a sufficient agreement is reached, which supports Equation (10).

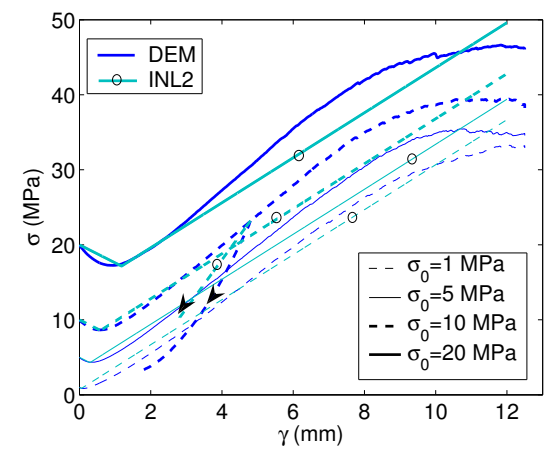

(a) $\sigma(\gamma)$

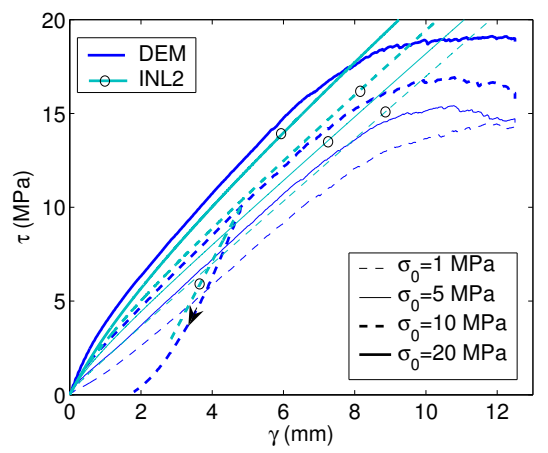

(b) $\tau(\gamma)$

Figure 5: Calibration of INL2 or Quadri relations for CND paths (here INL2=Quadri)

Second, Figure 6 presents the reference behaviour for the CTD paths. The numerical sample was subjected to a first CTD compression until a value of $\sigma \approx$ $10 \mathrm{MPa}$ was reached. It was then sheared under constant $\sigma$ until different values of $\gamma$ were obtained (the effects of these two first steps do not appear in Figure $6)$. From these different initial states, different CTD paths were simulated for loading $(d u>0)$ and unloading $(d u<0)$. The corresponding variations in $\sigma$ and $\tau$ are plotted on Figure 6.

Equation (11) proposes new definitions of the moduli corresponding to the 


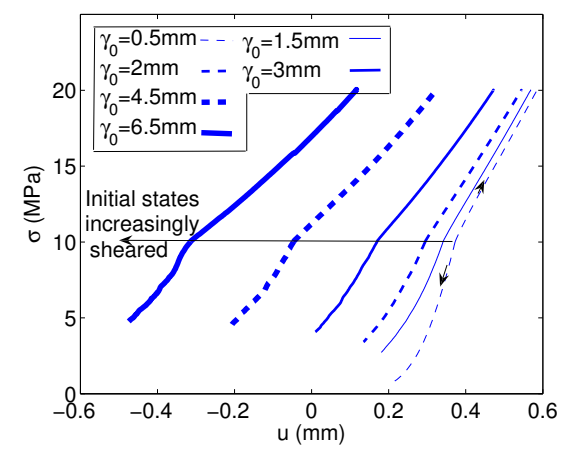

(a) $\sigma(u)$

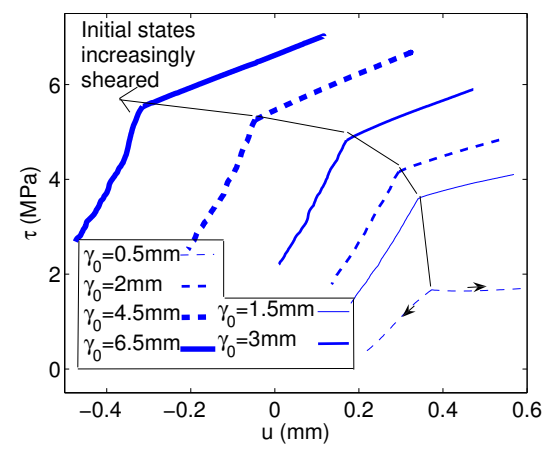

(b) $\tau(u)$

Figure 6: Reference CTD paths behaviour, from [2]

curves in Figure 6: $G_{u}^{+/-}$, and $N_{u}^{+/-}$.

$$
\begin{aligned}
N_{u}^{+} & =N\left(\frac{\tau}{\sigma}\right)\left(\frac{\sigma}{\sigma_{0}}\right)^{\frac{1}{3}} \\
& \text { with } N\left(\frac{\tau}{\sigma}\right)=\frac{20 N_{u}^{0}-N_{u}^{f}}{19}-\frac{N_{u}^{0}-N_{u}^{f}}{19} 20^{\frac{\tau / \sigma}{\tan (\varphi)}} \\
N_{u}^{-} & =N_{d} \times N_{u}^{+} \\
G_{u}^{+} & =\frac{G_{u}^{P}}{\tan (\varphi)} \frac{\tau}{\sigma}\left(\frac{\sigma}{\sigma_{0}}\right)^{\frac{1}{3}} \\
G_{u}^{-} & =\frac{G_{u}^{M}}{\tan (\varphi)} \frac{\tau}{\sigma}\left(\frac{\sigma}{\sigma_{0}}\right)^{\frac{1}{3}} \text { with } G_{u}^{M}=N_{d} \times N_{u}^{f} \times \tan (\varphi)
\end{aligned}
$$

In the case of normal stiffness $N_{u}^{+/-}$, the reference behaviour shows, consistent with experimental results [1, 7], an increase in the normal stiffness according to $\sigma$ and a decrease of this stiffness while shearing. Thus, the corresponding $N_{u}^{+}$ moduli is defined as increasing with respect to $\sigma$ and, through function $N(\tau / \sigma)$, decreasing according to $\tau / \sigma$. The use of the function $N$ leads to values of $N_{u}^{+}$, for $\sigma=\sigma_{0}$, equal to $N_{u}^{0}$ in a virgin state (with no shearing), and equal to $N_{u}^{f}$ if complete shearing is achieved $(\tau / \sigma=\tan (\varphi))$. Section 4 will indeed show that $\tan (\varphi)$ is the maximum value of the ratio $\tau / \sigma$. The corresponding unloading moduli $N_{u}^{-}$is taken to be proportional to $N_{u}^{+}$, through parameter $N_{d}$.

The $G_{u}^{+/-}$moduli are then defined as increasing with respect to both shearing (the $\tau / \sigma$ ratio), and normal stress $\sigma$. A parameter $G_{u}^{P}$ appears in the $G_{u}^{+}$ definition. It corresponds to the value of $G_{u}^{+}$under the completely sheared state: $\tau=\tau_{\max }=\sigma \tan (\varphi)$, for $\sigma=\sigma_{0}$. For the definition of $G_{u}^{-}$, a link is set between $G_{u}^{-}$and $N_{u}^{-}$, following section 2.2 .

Figure 7 shows both of the DEM results, and the INL2/Quadri relations (equations (2) and (6)) for such CTD paths. Good agreement is reached, which 
completes the calibration of our relations. The parameters and their retained

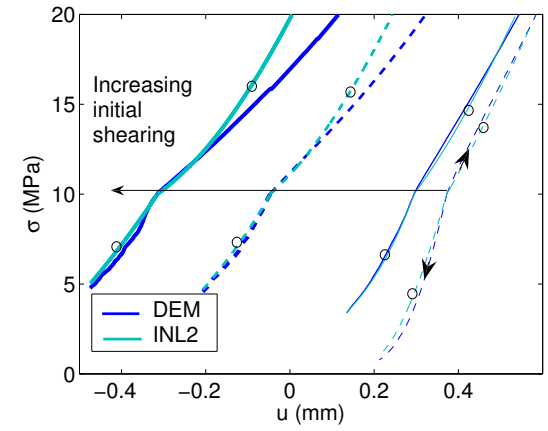

(a) $\sigma(u)$

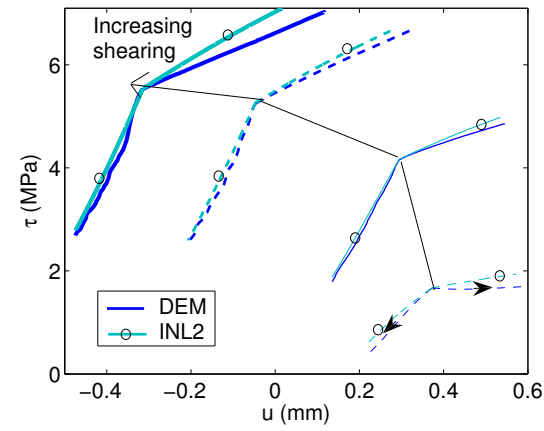

(b) $\tau(u)$

Figure 7: Calibration of INL2/Quadri relation for CTD paths (here INL2=Quadri)

values are summarised in Table 2 . We finally obtain nine independent parameters (the value of $\sigma_{0}$ is arbitrary and linked to the values of $N_{u}^{0}, N_{u}^{f}$ for example).

\begin{tabular}{|c|c|c|c|c|c|c|c|c|c|}
\hline \multicolumn{6}{|c|}{$(\mathrm{GPa} / \mathrm{m})$} & \multicolumn{2}{|c|}{$(\mathrm{MPa})$} & & \\
\hline$N_{\gamma}^{0}$ & $N_{\gamma}^{+}$ & $G_{\gamma}^{0}$ & $N_{u}^{0}$ & $N_{u}^{f}$ & $G_{u}^{P}$ & $N_{d}$ & $\sigma_{0}$ & $\varphi$ & $\varphi_{c}$ \\
\hline-2.4 & 3.0 & 3.6 & 20 & 8.0 & 2.4 & 2.0 & 1.0 & 29 & 12 \\
\hline
\end{tabular}

Table 2: INL2/Quadri relations parameters

\subsection{Validation}

To validate one or two of the constitutive relations, loading paths other than CND or CTD ones are considered. The DEM results of Duriez et al. [2] are still considered to be the reference. In this previous paper, the CNL and constant normal stiffness (CNS) paths were addressed. The corresponding results will be compared with the predictions of equations (2) and (6), with moduli such as those used here, in the equations (10) and (11).

The results of the discrete model of [2] and its predictions for the constitutive relations are compared in Figure 8 for CNL paths under different values of $\sigma$ : 1, 5, 10 and $20 \mathrm{MPa}$.

Concerning the $\tau(\gamma)$ curve, Figure 8(a) shows that the INL2 and Quadri predictions are close to each other, and to reference discrete results. In the case of $u(\gamma)$ in Figure 8(b), the differences between the two predictions appear. 


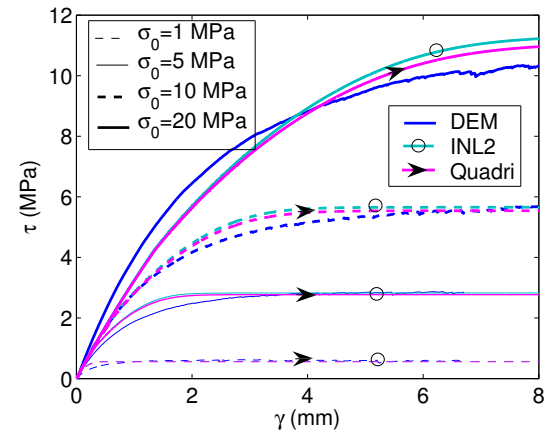

(a) $\tau(\gamma)$

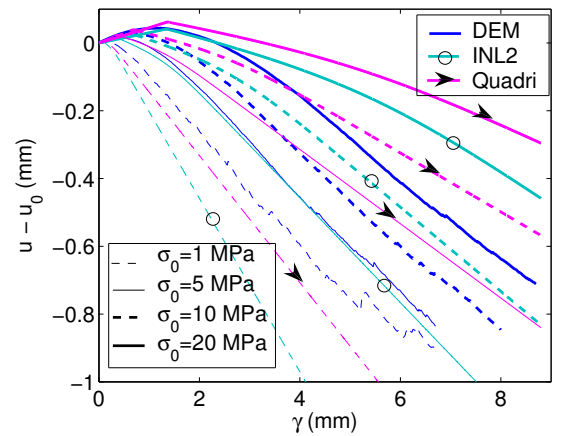

(b) $u(\gamma)$

Figure 8: Predictions of the constitutive relations, towards discrete results (from [2]) for CNL loading paths

Compared with DEM, the INL predictions are very efficient for middle values of $\sigma(5$ or $10 \mathrm{MPa})$, and a little less for extreme values of $\sigma(1$ or $20 \mathrm{MPa})$. The Quadri predictions are further from those of DEM, with the exception of the $\sigma=1 \mathrm{MPa}$ case. In general, the Quadri predictions correspond to a less dilatant behaviour than do the INL ones.

CNS paths are then considered. Such loadings are shearings for which attention is paid both to changes in $\sigma$ and $u$. The test is then monitored so that $d \sigma / d u=\chi$ during the entire test, where $\chi$ is a chosen constant. Figure 9 shows the comparison between the discrete results and the INL2 or Quadri predictions for three different CNS tests. The three tests start from the same value of $\sigma$ (5 MPa) but correspond to different values of $\chi: 10,40$ and $120 \mathrm{MPa} / \mathrm{mm}$. Again, the INL2 behaviour here is more dilatant than that of the Quadri (see the $\sigma(\gamma)$ and $u(\gamma)$ curves in Figures 9(c) and 9(b)) and the INL2 predictions are still generally more efficient than the Quadri ones, even if the differences are not as important. As differences would be especially so small in Figure 9(d), the Quadri results are not plotted in this subfigure so that the curves will remain readable. Finally, these results lead us to consider our constitutive relations to be reasonably validated.

Now that calibration and validation have been performed, a plasticity analysis of the two relations will be considered, focusing first on plasticity limit criterion. 


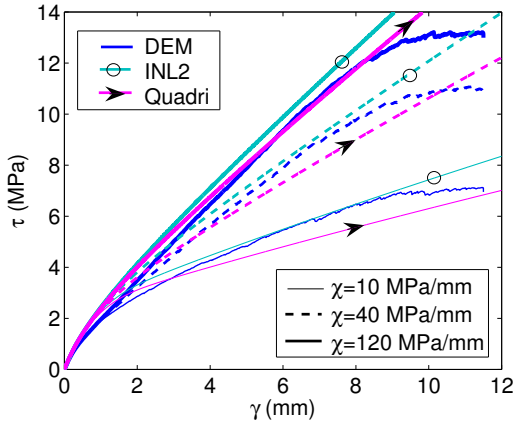

(a) $\tau(\gamma)$

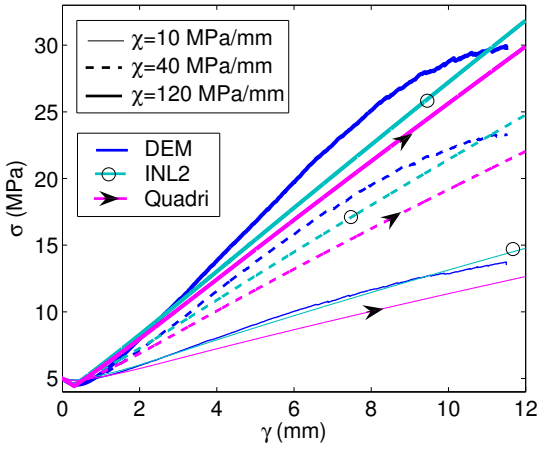

(c) $\sigma(\gamma)$

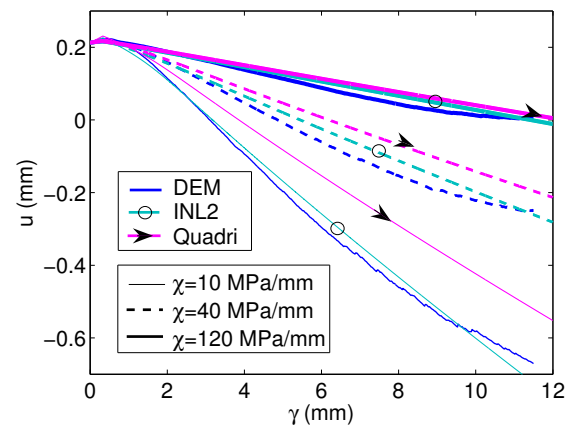

(b) $u(\gamma)$

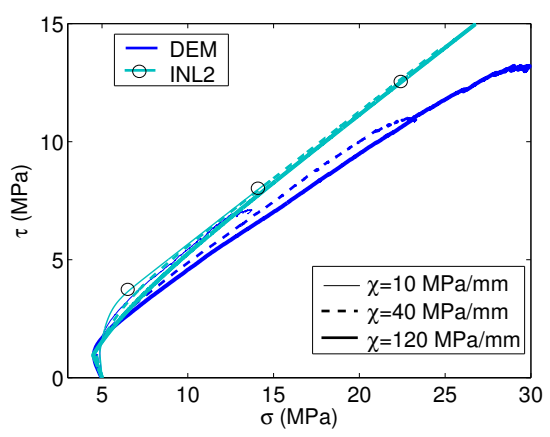

(d) $\tau(\sigma)$

Figure 9: Predictions of the relations, towards discrete results (from [2]) for CNS loading paths 


\section{Plasticity limit criterion of these constitutive relations}

Following the considerations of section 2.2 , the existence of a plasticity limit criterion is discussed. A parameter $\varphi$ was introduced in the expressions for some of the moduli. This section will show that this parameter corresponds to a friction angle in a Mohr-Coulomb criterion that limits the stress states described by our relations. The Quadri and INL2 cases will be successively debated. The existence of the plasticity limit criterion for the discrete model was discussed previously [2].

\subsection{Quadri case}

Let us assume a stress state located on this supposed Mohr-Coulomb criterion: $(\sigma, \tau=\sigma \tan (\varphi))$. From this state, any loading $\overrightarrow{d l}$ is considered and it is defined by its direction $\theta d l$ (see Figure 10(a)). The direction of the corresponding response $\overrightarrow{d \sigma}$ is denoted by $\theta d \sigma$ (see Figure $10(\mathrm{~b})$ ). Due to Equation (1), the

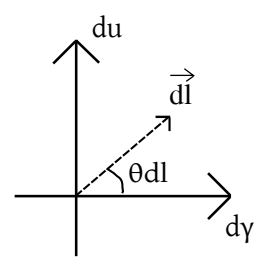

(a)

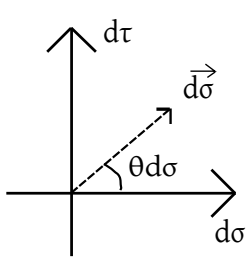

(b)

Figure 10: Definition of angles $\theta d l$ (in displacement plane) and $\theta d \sigma$ (in stress plane)

direction $\theta d \sigma$ depends only on the direction $\theta d l$. The Mohr-Coulomb criterion would then be violated if there exists some $\theta d l$ leading to $\theta d \sigma \in\left[\varphi ; \varphi+180^{\circ}\right]=$ $\left[29^{\circ} ; 209^{\circ}\right]$, in our case where $\varphi=29^{\circ}$ (see Table 2).

Figure 11 examines this question. Thanks to the use of Quadri relation, Equation (6), $\theta d \sigma$ can be computed according to $\theta d l$. For the stress states of the Mohr-Coulomb criterion, the corresponding relation still depends on $\sigma$, and the two values ( 1 and $10 \mathrm{MPa}$ ) are thus considered in Figure 11. Figure 11 shows that values of $\theta d \sigma$ between $\varphi=29^{\circ}$ and $180+\varphi=209^{\circ}$ are never reached. The plasticity limit criterion defined by $\tau_{\max }=\sigma \tan (\varphi)$ seems here to be never violated. Figure 11 moreover illustrates that there are some loading directions that lead to the responses following the Mohr-Coulomb criterion; these loadings all implying the compression unloadings $d u<0\left(\theta d l \in\left[180^{\circ} ; 360^{\circ}\right]\right)$. The discrete results of Duriez et al. [2] (see also Figure 23) have indeed shown that the CTD unloadings $\left(\theta d l=270^{\circ}\right)$ from completely sheared states lead to decreases in the stresses, with the stress states remaining consinstent with the Mohr-Coulomb criterion, whereas any loading withthe compression $d u>0(\theta d l \in] 0^{\circ} ; 180^{\circ}[)$ 


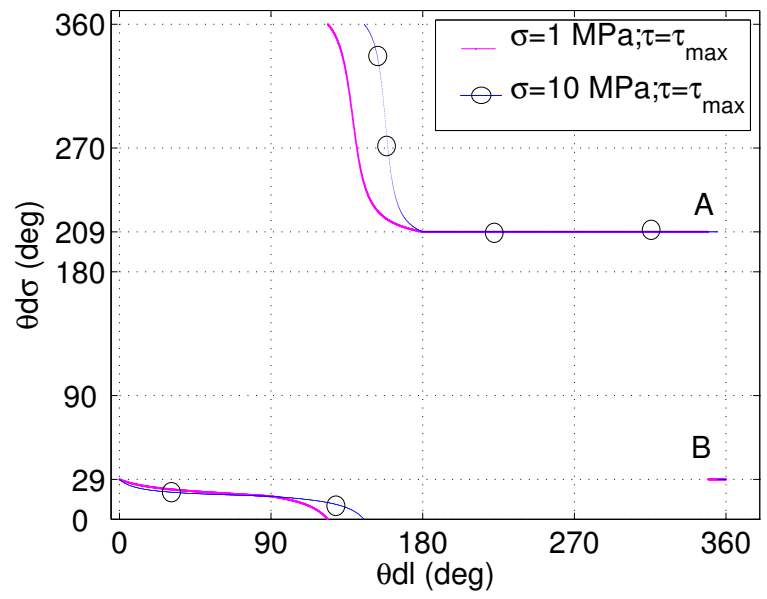

Figure 11: Quadri case: link between displacement loading, and stress response directions. For two stress states on plasticity limit criterion. AB discontinuity is linked to the existence of a plastic limit criterion of Mohr-Coulomb type

carries the stress state strictly inside the plasticity limit criterion.

The properties of the function $\theta d \sigma=f(\theta d l)$ are linked with the cancellation of the determinants of the constitutive matrices, as described in section 2.2. Figure 12 shows the evolution of the determinants of the four matrices from the Quadri case. The determinants are generally decreasing (from their initial positive values) during the shearing. A discontinuous change is observed that corresponds to the discontinuous change of the $N_{\gamma}^{+/-}$moduli at the contractantdilatant transition. Among the four matrices, the two corresponding to the tensorial zones with $d u<0$ vanish exactly on the plasticity limit criterion. Compared with Figure 3, which considered other expressions for the moduli, the cancellations no longer occur before the plasticity limit criterion. In fact, as the matrices are defined inside a tensorial zone, it must be verified that the eigenvectors of the eigenvalue 0 (obtained due to the determinant cancelling) belong to the correct tensorial zone - we note already that such eigenvectors correspond to flow rule, as it will be discussed in section 6 . This verification is performed in Figure 13. In this way, we verify that the eigenvectors of eigenvalue 0 do not belong to the right tensorial zone for $(d \gamma<0, d u<0)$, as they correspond to $(d \gamma>0, d u<0)$ or $(d \gamma<0, d u>0)$. Hence, the corresponding matrix for this tensorial zone is finally not to be considered with a vanishing determinant. By contrast, cancellation of the determinant of the corresponding matrix is confirmed for $(d \gamma>0, d u<0)$. The eigenvectors of the matrix belong to the tensorial zone in which the matrix exists.

Due to the expressions for moduli in equations (10) and (11), such cancella- 


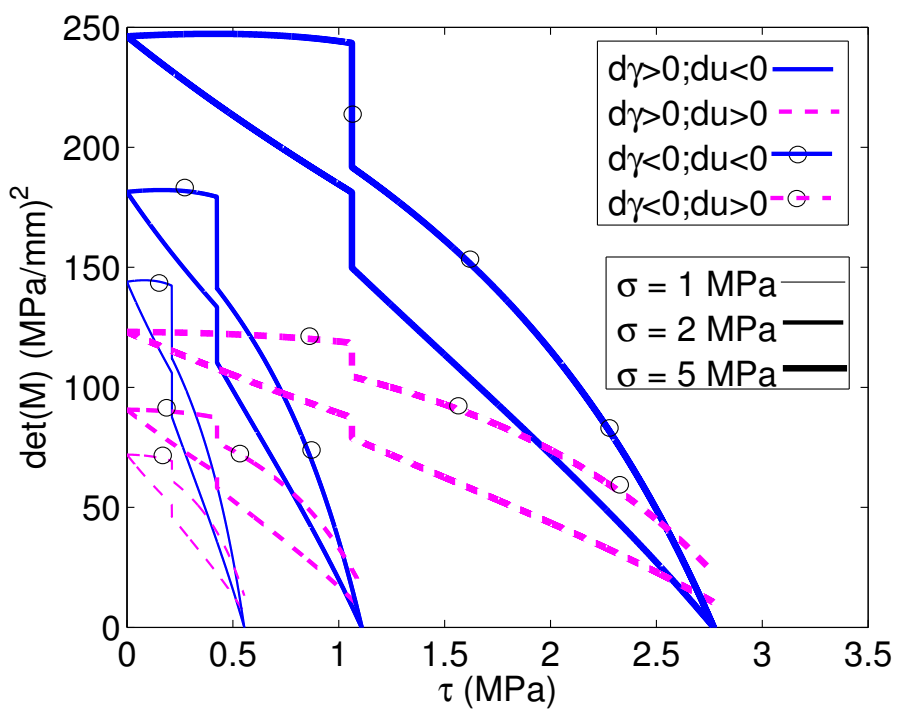

Figure 12: Quadri case: determinants of constitutive matrices according to $\tau$ for different values of $\sigma$

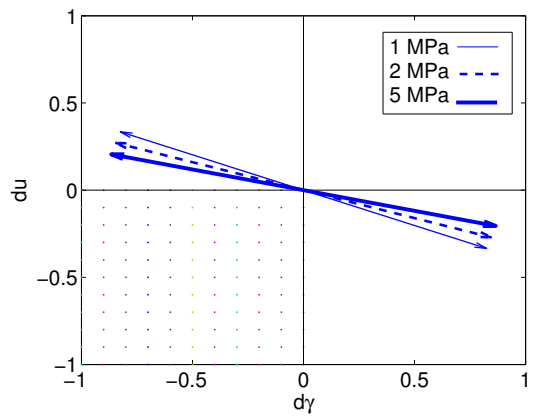

(a) $(d \gamma<0, d u<0)$ tensorial zone

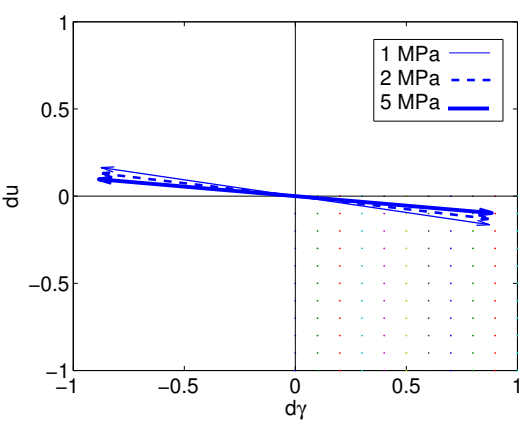

(b) $(d \gamma>0, d u<0)$ tensorial zone

Figure 13: Eigenvectors of eigenvalue 0, for both matrices corresponding to $d u<0$ (with $d \gamma>$ or $<0$ ). For this vanishing eigenvalue, directions have to be considered, not only vectors 
tions are obtained for any value of $\sigma$. As we obtain in our case, on the plasticity limit criterion:

$$
\begin{aligned}
\operatorname{det}\left(M^{+-}\right) & =G_{\gamma}^{+} N_{u}^{-}-N_{\gamma}^{+} G_{u}^{-}=G_{\gamma}^{f} N_{d} N_{u}^{f}\left(\frac{\sigma}{\sigma_{0}}\right)^{\frac{1}{3}}-N_{\gamma}^{+}{ }_{d i l} G_{u}^{M}\left(\frac{\sigma}{\sigma_{0}}\right)^{\frac{1}{3}} \\
& =\left(N_{\gamma_{d i l}^{+}}^{+} \tan (\varphi) N_{d} N_{u}^{f}-N_{\gamma_{d i l}}^{+} N_{d} N_{u}^{f} \tan (\varphi)\right)\left(\frac{\sigma}{\sigma_{0}}\right)^{\frac{1}{3}}=0 \forall \sigma
\end{aligned}
$$

These results prove the existence of a plasticity limit criterion (of the MohrCoulomb type without cohesion) outside of which stress states cannot be obtained with our Quadri relation. This criterion is not directly stated, but appears through the retained expressions for the moduli. The definitions allow the required cancellations of the determinants of the constitutive matrices in the criterion. Moreover, our results illustrate the fact that limit stress states can be obtained only with a shear loading $(d \gamma \geq 0)$ that is linked with dilatancy $(d u<0)$. Whereas other loadings (e.g., loading compressions $d u>0)$ move the rock joint stress state further inside the plasticity limit criterion. This result is consistent with a nonvanishing determinant of the constitutive matrix (see also next section 6.5).

\subsection{INL2 case}

For the INL2 case, such an analysis using piecewise constant constitutive matrices cannot be performed. However, stress states also seem to be limited in this case: see for example Figure 8(a), in which the INL2 $\tau(\gamma)$ curves for the CNL tests also level off (without any correction). The threshold obtained is close to the one for the Quadri curves. In addition to the CNL tests considered in Figure 8, nine other CNL tests were carried out with INL2 relation using other $\sigma$ values in the range $[1 \mathrm{MPa} ; 20 \mathrm{MPa}]$. For all of these CNL tests, we observe that the maximum value of $\tau, \tau_{\max }$, corresponds to values of $\arctan \left(\tau_{\max } / \sigma\right)$ remaining in the range $\left[29.4^{\circ} ; 29.5^{\circ}\right]$.

To determine if this condition remains valid for any loadings other than the CNL ones, we perform the same analysis that led to Figure 11. The final stress states (i.e. $\tau$ thresholds) for the CNL loadings under 1 and $10 \mathrm{MPa}$ (performed using the INL2 relations) are considered. From these mechanical states, a displacement probe test is performed in the INL2 framework. The stress response direction $\theta d \sigma$ can then be plotted according to $\theta d l$ in Figure 14. The major point of Figure 14 is that there is also no stress response increment for the INL2 case in the $\left[29^{\circ} ; 209^{\circ}\right]$ range, which would bring the stress state outside the plasticity limit criterion. We note that there might be an approximately $0.5^{\circ}$ error on these values, as the value of the observed friction angle in the INL2 case is approximately $29.5^{\circ}$ rather than exactly $29^{\circ}$.

Finally, the existence of a plasticity limit criterion is also obtained for the INL2 relation. It can be considered to be identical to the one for the Quadri

relation. Both are obtained from the moduli expression. Thanks to the response 


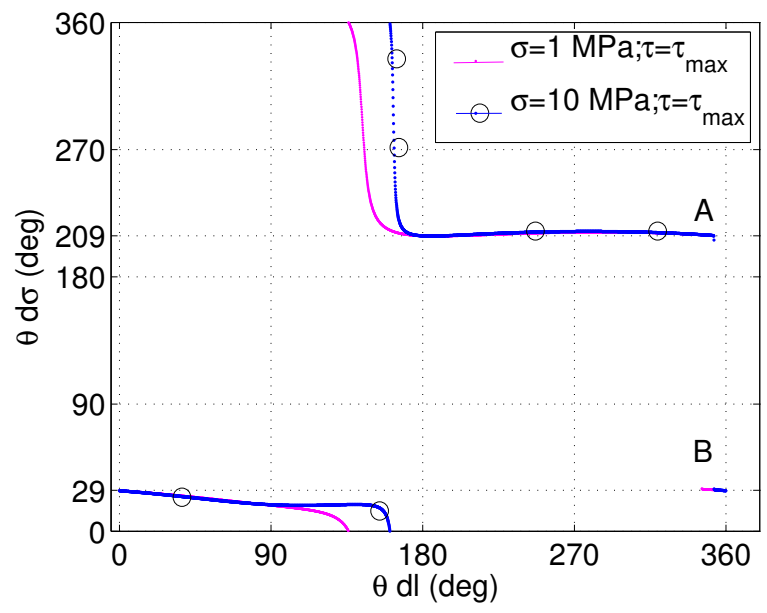

Figure 14: INL2 case: link between displacement loading, and stress response directions. For two stress states. AB discontinuity is linked to the existence of a plastic limit criterion of Mohr-Coulomb type

envelopes, following section 5 illustrates the changes in the rock joint behaviour when the plastic limit criterion is approached.

\section{$5 \quad$ Response envelopes}

Response envelopes studies [34] allow checking the consistency of proposed constitutive equations [20]. Indeed, in the case of incrementally piecewise linear constitutive relations, the elasto-plastic matrix is changing suddenly from one tensorial zone to another (we recall that, by definition, the constitutive relation inside a tensorial zone is linear and thus characterised by a given matrix). From physical and experimental points of view, however, the material response must be continuous when the loading direction is passing through the border between two tensorial zones. This condition is the so-called "continuity condition" (see [4] for details). For elasto-plastic constitutive relations, it is possible to demonstrate that the continuity condition is always fulfilled if the consistency condition of the elasto-plastic theory is properly written. In this paper, however, the constitutive relations have not been developed in a classical elasto-plastic framework, and thus, the continuity condition must be specifically verified.

From an analytical point of view, we consider the Quadri case, Equation (6), and Table 1. As an example, we focus on the tensorial zone $(d \gamma<0, d u>0)$, with the elasto-plastic matrix $M$ equal to $M^{-+}$, and the tensorial zone $(d \gamma>$ $0, d u>0)$ with the $M^{++}$matrix. The frontier between these two zones is the half straight line in the $(d \gamma, d u)$ plane whose equation is $(d \gamma=0, d u>0)$. The first column of the $M$ matrix is changing through this frontier, from $M^{-+}$to 
$M^{++}$. In a consistent manner, this first column is multiplied by $d \gamma=0$ to determine the material response, which is thus perfectly continuous.

This analytical reasoning can be graphically confirmed by considering the response envelopes that illustrate the material response vectors $(\overrightarrow{d \sigma}$ or $\overrightarrow{d l})$ for a given state by considering the loading vectors $(\overrightarrow{d l}$ or $\overrightarrow{d \sigma}$ ) in all the directions $\theta d l$ or $\theta d \sigma$ (see previous Figure 10). Hence, the response envelopes are presented for our relations. Comparisons are performed between the Quadri and INL2 cases. Then the influences of the normal stress value and shearing state are investigated. The shearing state is described by the variable $\phi_{m o b}=\arctan (\tau / \sigma)$. No shearing corresponds to $\phi_{m o b}=0^{\circ}$, and complete shearing corresponds to $\phi_{m o b}=\varphi=29^{\circ}$ (see section 4). The displacement and stress probes are imposed, in both cases 3600 incremental displacement loadings are considered, resulting in $\theta d l$ or $\theta d \sigma$ covering $\left[0^{\circ} ; 360^{\circ}\right.$ ], by steps of $0.1^{\circ}$. The norms of the stress or displacement probes $\left(\|\overrightarrow{d \sigma}\|=100 \mathrm{kPa}\right.$, or $\left.\|\overrightarrow{d l}\|=10^{-5} \mathrm{~m}\right)$ are arbitrary and do not affect the shape of the envelopes here.

\subsection{Comparison between Quadri and INL2 cases}

A comparison between the envelope responses of our two relations is shown in Figure 15. For this Figure, the displacement probes, from two different mechanical states, are considered. A constant value for $\sigma(10 \mathrm{MPa})$ is used, but two different values of $\tau$, i.e., two different values of $\phi_{m o b}$, are considered. This Figure 15 shows that the Quadri and INL2 relations have similar response

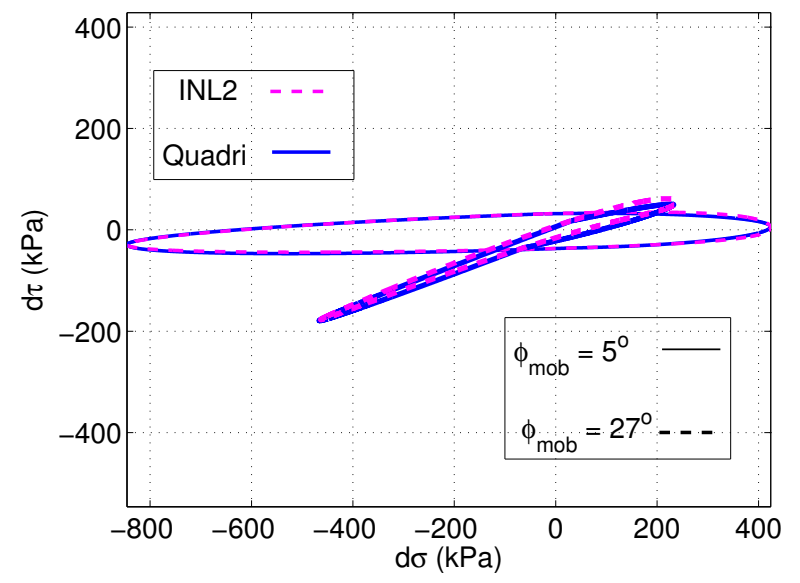

Figure 15: Comparison of envelope responses for INL2 and Quadri relations; for $\sigma=10 \mathrm{MPa}$, and two different values of $\tau$

envelopes. Slight differences appear only for the sheared states of the joint. For this reason, only the INL2 relation is considered in following sections 5.2 and 5.3 . 
We remark that an elastic behaviour is characterised by an ellipse whose centre corresponds to the plane origin $(d \sigma=0, d \tau=0)$. For an incrementally piecewise linear elasto-plastic relation, the response envelope is constituted by arcs of ellipses that form a continuous diagram if the constitutive relation is continuous. The elasto-plastic natures of the proposed constitutive relations and their continuities are clearly visible in Figure 15.

As a comparison with previous similar relations, Figure 16 uses data from [20] to compare the response envelopes of two INL2 and Octo relations that were calibrated on soils. We recall that the Octo relation corresponds to the Quadri relation, but in 3D (it then presents eight tensorial zones). In [20], stress probes were performed under axisymmetric conditions, from different stress states $\left(\sigma_{1} ; \sigma_{2}=\sigma_{3} ; \sigma_{3}\right)$. In this case also, the differences between Octo

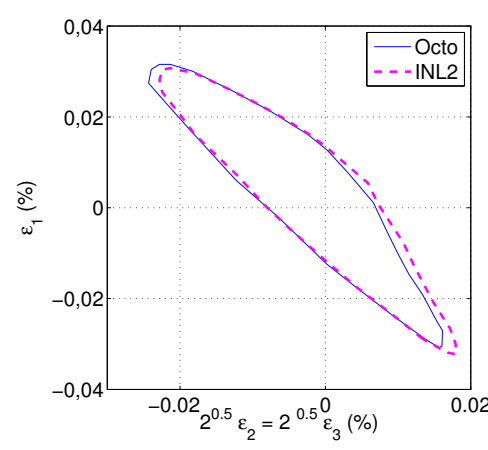

(a) $\sigma_{1}=\sigma_{3}=100 \mathrm{kPa}$

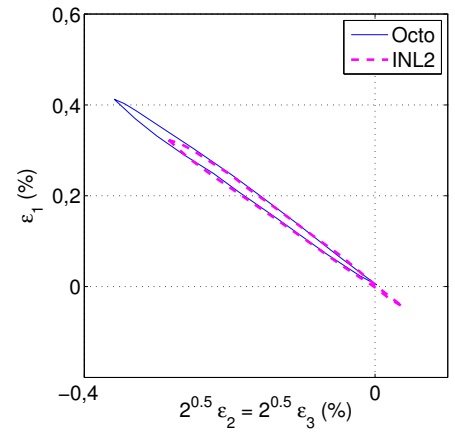

(b) $\sigma_{1}=400 \mathrm{kPa} ; \sigma_{3}=100 \mathrm{kPa}$ (near failure)

Figure 16: Comparison of response envelopes for simulations with Octo and INL relations, for soils' case. After data of [20]

(which is thus compared to the Quadri case) and INL2 relations appear significant only if the sample is sufficiently sheared. The differences in this case are greater than in the case of the rock joints relations.

\subsection{Influence of $\sigma$}

Figure 17 considers four response envelopes of the INL2 relation for four different mechanical states, with two different values of $\sigma$, under two shearing states (two different $\left.\phi_{m o b}\right)$. Whatever the shearing state, increasing values of $\sigma$ change only the size of the response envelopes and not their shape.

\subsection{Influence of shearing}

The influence of previous shearing on the response envelopes is then studied alone, with a constant value for $\sigma(10 \mathrm{MPa})$. Figure 18 considers displacement and stress probes for several $\phi_{m o b}$ values. Contrary to the displacement case, 


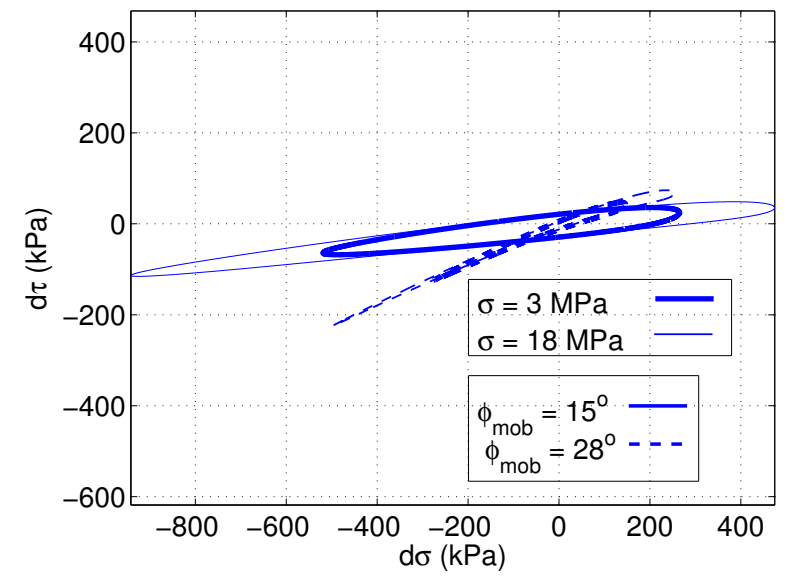

Figure 17: INL2 response envelopes: influence of $\sigma$

no stress probe can be performed under $\phi_{m o b}=29^{\circ}$. Indeed, section 4.2 showed that, for these mechanical states, the set of stress directions $\theta d \sigma$ directed outside the Mohr-Coulomb limit line is never reached, for any value of $\theta d l$. These directions cannot then be considered, and complete stress probe cannot be executed. That being said, the evolutions of the stress response envelopes during shearing

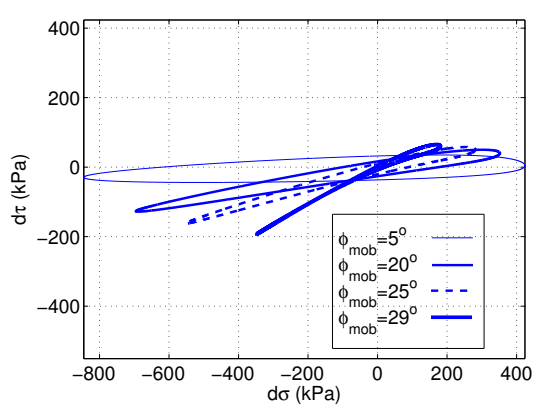

(a) Displacement probes

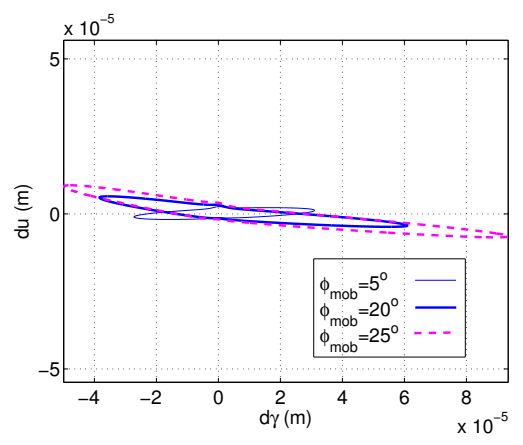

(b) Stress probes

Figure 18: INL2 response envelopes: influence of shearing (for $\sigma=10 \mathrm{MPa}$ )

appear clearly in Figure 18(a). From an initial curve that is close to an ellipse, the stress envelopes change until presenting, under the Mohr-Coulomb criterion, a straight section that corresponds to the plasticity limit criterion $\left(\theta d \sigma=209^{\circ}\right.$ for this straight section). The same trends can be observed for the displacement response envelopes in Figure 18(b), with the envelopes particularly growing in the flow rule direction as the shearing increases. 


\section{Flow rules of the relations and the discrete model}

When a limit stress state is reached on the plastic limit criterion $(\|\overrightarrow{d \sigma}\|=\overrightarrow{0}$, e.g., CNL shearing at the end of the shearing), the relative displacements still evolve. However, their direction is fixed by the flow rule of the rock joint. A precise definition of this flow rule will be proposed before it is applied to our relations and our discrete model. Comparisons and concluding remarks about nonassociativity and singularity of the flow rule are then presented.

\subsection{Definition of the flow rule}

Classical elasto-plastic relations define elastic and plastic parts of strains (relative displacements in rock joints case). In this framework, plastic strains, once they appeared, have a direction imposed by the material flow rule. The Quadri and INL2 relations do not distinguish plastic or elastic strains (relative displacements). Nevertheless, we can define a flow rule from the displacement increments that occur when the stresses reach the plastic limit criterion: the vector $d \vec{l} p=\left(d u^{p}, d \gamma^{p}\right)$. As discussed by Darve \& Nicot [35], this definition is close to the classical one, as on the plastic limit criterion, the elastic part of the deformation becomes negligible. In our case, the flow rule controls the ratio $d u^{p} / d \gamma^{p}$ through a dilatancy angle $\psi$, such that:

$$
\tan (\psi)=-\frac{d u^{p}}{d \gamma^{p}}
$$

Figure 19 illustrates this definition. The $(\sigma, \tau)$ and $(u, \gamma)$ planes are superposed

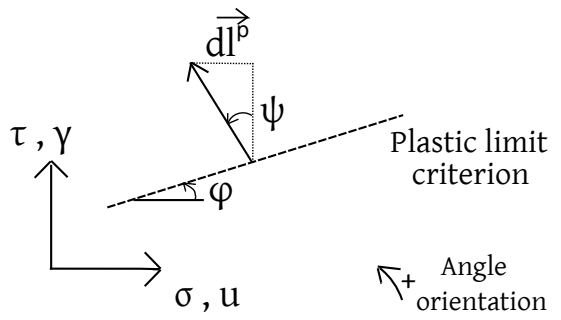

Figure 19: Rock joint flow rule: illustration

on the figure, as it is required when flow rules are tackled. The angle $\psi$ can be measured from the $\tau$ (or $\gamma$ )-axis to the vector $d \vec{l}^{p}$. For $\psi=\varphi$, we verify that we would obtain an associated behaviour: $\overrightarrow{d l^{p}}$ would be orthogonal to plastic limit criterion.

The dilatancy angle $\psi$ can, for example, be observed in Figure 8(b) as the angle between the $u(\gamma)$ curves and the $\gamma$-axis, for the section of the curves corresponding to the $\tau$ plateau. 


\subsection{Flow rule for Quadri case}

Linear expressions in the Quadri case allow the flow rule to be easily determined. We solve equation $d \sigma=M \overrightarrow{d l}=\overrightarrow{0}$, with $\overrightarrow{d l}$ unknown and $M$ such that $\operatorname{det}(M)=$ 0 (the plastic limit criterion) and determine the direction of the set of solutions that is obtained in this case. This is performed in following Equation (14), in which moduli of the $(d \gamma>0, d u<0)$ tensorial zone are considered. Section 4.1 showed that the vectors $\overrightarrow{d l}$ from the other quadrants cannot be solutions to this equation, implying that the flow rule belongs to the $(d \gamma>0, d u<0)$ tensorial zone.

$$
\begin{aligned}
& \left\{\begin{array}{c}
d \tau=G_{\gamma}^{+} d \gamma+G_{u}^{-} d u=0 \\
d \sigma=N_{\gamma}^{+} d \gamma+N_{u}^{-} d u=0
\end{array} \Leftrightarrow \frac{d u}{d \gamma}=-\frac{G_{\gamma}^{+}}{G_{u}^{-}}=-\frac{N_{\gamma}^{+}}{N_{u}^{-}}\right. \\
& \Leftrightarrow \tan (\psi)=\frac{G_{\gamma}^{+}}{G_{u}^{-}}=\frac{N_{\gamma}^{+}}{N_{u}^{-}}
\end{aligned}
$$

Obviously, equality of the last two terms in Equation (14) corresponds to cancellation of the determinant. With the expressions for the moduli proposed in equations (10) and (11) we finally obtain:

$$
\tan (\psi)=\frac{N_{\gamma d i l}^{+}}{N_{d} N_{u}^{f}\left(\sigma / \sigma_{0}\right)^{1 / 3}}
$$

Such a flow rule is plotted according to $\sigma$ in Figure 22 (appearing with other results on page 26). Equation (15) indicates already that the dilatancy angle $\psi$ decreases according to $\sigma$ and to a parameter linked with normal rigidity $\left(N_{u}^{f}\right)$,

whereas increasing the dilatant feature of the joint $\left(N_{\gamma}^{+}\right.$dil parameter $)$allows $\psi$ to increase.

\subsection{Flow rule for INL2 case}

For the INL2 case, the previous analytical development is no longer possible. However, the values of $\psi(\sigma)$ can be measured in two ways.

First, the slopes of $u(\gamma)$ curves for the CNL tests, once on the $\tau$ plateaus, are measured from results presented in Figure 8(b). As explained before, such slopes depend directly on $\psi$ through equation (13).

An other method for determining $\psi$ value leads to the same results. It relies on "proportional displacement loading paths", that are performed using INL2 relation. Such paths are governed by $d u / d \gamma=R \Leftrightarrow d u-R d \gamma=0$.

To understand the behaviour for such paths on the plasticity limit criterion, it is only necessary to compare the volume variation rate imposed by the loading path (through the constant $R$ ) to the material volume variation rate (which is issued from the dilatancy angle, $\psi$ ). If the loading path volume variation rate is higher (i.e., more dilatant) than the material volume variation rate, stresses decrease along or close to the descending branch of the plasticity limit criterion. When the stresses are decreasing, the material dilatancy angle should decrease. 
If it reaches the imposed volume variation rate, an asymptotic stress state is met and failure will develop: strains still evolve while the stresses remain constant. If the dilatancy angle does not reach the imposed rate before the stresses vanish, liquefaction will develop (see [36] for a general liquefaction criterion). On the contrary, if the imposed volume variation rate is lower (i.e., more contractant or less dilatant) than the material volume variations rate, stresses increase along or close to the ascending branch of the Mohr-Coulomb criterion. The dilatancy angle is decreasing along with this stress increase. When the related volume variation rate is equal to the imposed volume variation rate, an asymptotic stress point is again reached, accompanied by failure.

We use this consideration to determine the value of $\psi$ for the INL2 case. Different values of $R$ are chosen, and for each value, different initial stress states are considered. Examples of the results of the test for $d u / d \gamma=-\tan \left(10^{\circ}\right)$ appear in Figure 20. The curves of Figure 20 show that, regardless of the initial
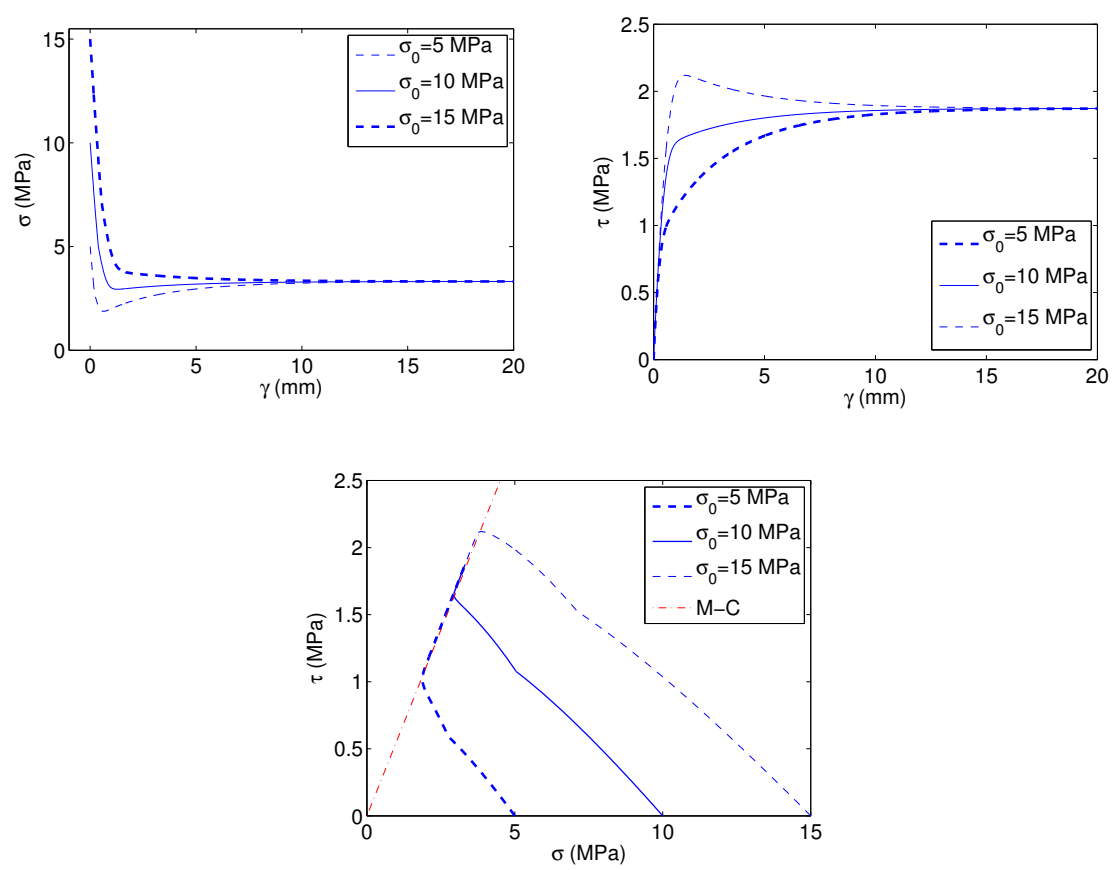

Figure 20: Stresses evolutions for proportional displacement loading with $d u / d \gamma=-\tan \left(10^{\circ}\right)$, from three initial stress states (INL2 constitutive relation)

state, the stresses reach the Mohr-Coulomb criterion during this slightly dilatant shearing path. Then, after some additional changes (increases or decreases, depending on the previous discussion) in stresses on the criterion, a final stress point is reached: $\tau$ and $\sigma$ do not evolve any further. As explained above, this 
case occurs when the loading parameters correspond to the flow rule of the INL2 relation (its existence is also proved by these results). For other values of $R$, another final stress point, still on Mohr-Coulomb criterion but with a different value of $\sigma$, is reached: as for Quadri case, the dependence of the dilatancy angle on $\sigma$ is obtained here in INL2 case. For example, the results in Figure 20 show that $10^{\circ}=\psi(\sigma \approx 3.3 \mathrm{MPa})$. The collected values of $\psi(\sigma)$ for the INL2 relation will finally be plotted in Figure 22 (on page 26).

As the values of $\psi(\sigma)$ for the INL2 and Quadri relations are not exactly equal (a comparison appears in Figure 22), predictions of these two relations show some differences near the plastic limit criterion along these proportional displacement loading paths, see Figure 21. With both relations, four paths are

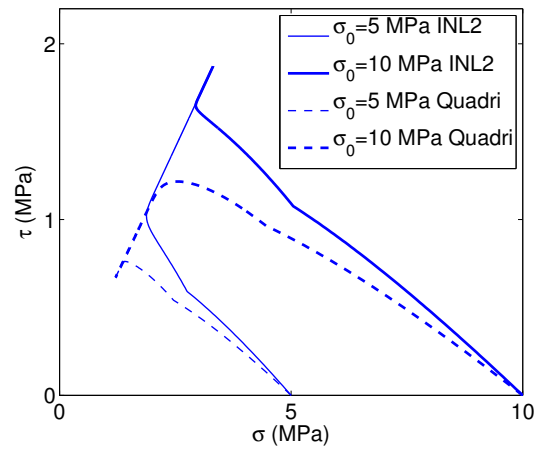

Figure 21: Proportionnal displacement loading paths $\left(d u / d \gamma=-\tan \left(10^{\circ}\right)\right)$ for Quadri and INL2 relations, from two different initial stress states. For each relation both curves reach the same final state for which flow rule corresponds to loading parameter: $\psi(\sigma)=10^{\circ}$

imposed for the same control parameter, $R=-\tan \left(10^{\circ}\right)$, from two different initial stress states. As explained earlier, all the curves converge on the stress states of the Mohr-Coulomb plastic criterion, for $\sigma$ values such that $\psi(\sigma)=10^{\circ}$. And the differences in the $\psi(\sigma)$ values for both relations lead here to different behaviours on plastic criterion. In this case, we obtain on the criterion $d \sigma<0$ for the Quadri-performed paths (a contractant behaviour), and $d \sigma>0$ for the INL2-performed paths (a dilatant behaviour), whereas the differences are smaller away from the criterion.

\subsection{Flow rule for DEM simulations}

Reference [2] discussed the plasticity limit criterion of the used discrete numerical model for infilled rock joints, without focusing on flow rule. Nevertheless, the discrete results allow also to point out this feature, in at least two ways. 
First, the final slopes of the $u(\gamma)$ curves for the CNL tests (Figure 8), once on the plateau of $\tau$, are measured and the corresponding values of $\psi$ are deduced. They are plotted according to $\sigma$ in same Figure 22. Errors (which are caused by nonlinearity of discrete $u(\gamma)$ curves in Figure 8(b)) for these values are plotted as error bars. For the two values corresponding to $\sigma=10$ or $20 \mathrm{MPa}$, the bars are in fact contained in the marker that is used. Figure 22 gathers the evolutions of $\psi$ with $\sigma$ according to the three different models used: the Quadri and INL2 relations, and the discrete numerical model. All the models present a dilatancy angle that decreases with $\sigma$, and the values of $\psi$ for the different models are close. The main point is that the dilatancy angle is clearly different from the friction angle (which is here equal to $29^{\circ}$ ): the rock joint behaviour, approached from the three different perspectives, is nonassociated.

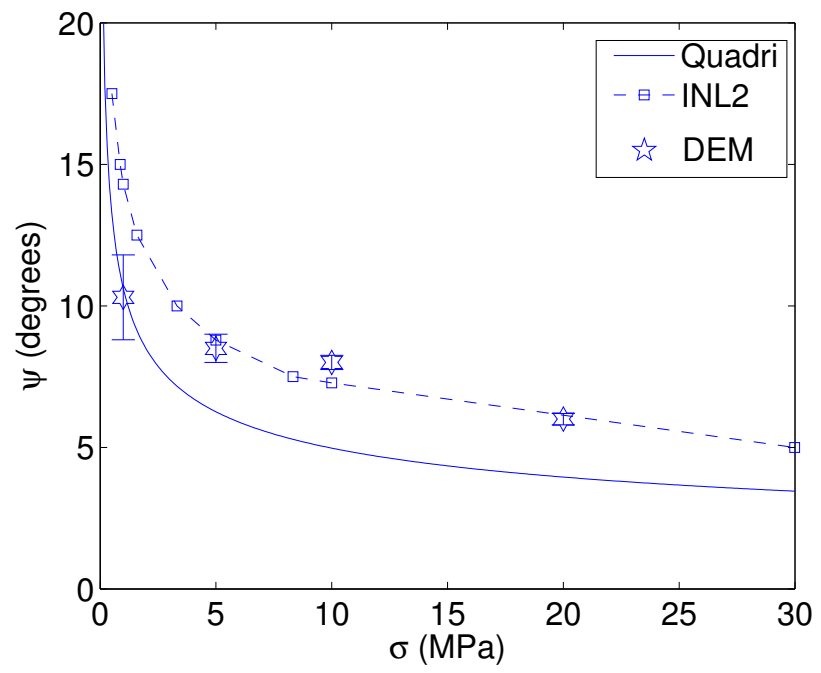

Figure 22: Evolution of $\psi$ depending on $\sigma$, a comparison between Quadri and INL2 constitutive relations, and DEM results

Second, other discrete results indicate the flow rule. Similar to what was done with INL2 relation, different proportional displacement loading paths are performed with the discrete model. The tests are governed by parameter $\theta d l$ : we impose the condition $d u-d \gamma \tan (\theta d l)=0(R=\tan (\theta d l))$. All the tests are performed from the mechanical state reached at the end of a CNL test under $\sigma=10 \mathrm{MPa}$. The corresponding changes in stress appear in Figure 23. Figure $23(\mathrm{a})$ shows that the tests for $\theta d l \in\left[-5^{\circ} ; 15^{\circ}\right]$ allow the stresses to increase, while the tests with $\theta d l \in\left[-20^{\circ} ;-5^{\circ}\right]$ allow the stresses to decrease. As Figure 23(b) clearly shows, we can assume that for $\tan \left(-10^{\circ}\right)<d u / d \gamma<\tan \left(-5^{\circ}\right)$, the stresses would not evolve and that the flow rule would be obtained. This result confirms the previous discrete results, which showed already that $5^{\circ}<\psi(\sigma=$ 


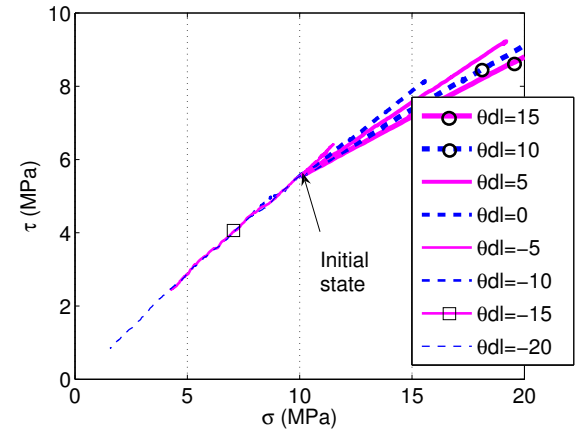

(a) In Mohr plane

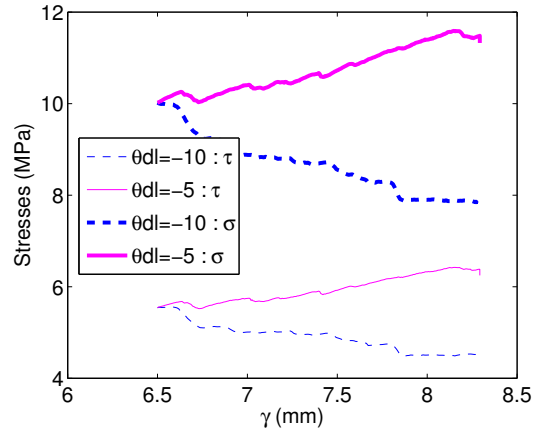

(b) According to $\gamma$, for loading parameters around the flow rule

Figure 23: Stress changes for different proportional displacement loading paths (with $d u / d \gamma=\tan (\theta d l)$ ), from a completely sheared state

$10 \mathrm{MPa})<10^{\circ}$.

The discrete results in Figure 23(a) results can also be compared with those Figures 11 and 14. As in the Quadri and INL2 cases, the loading paths from the Mohr-Coulomb criterion with $d u>0$ move the rock joint stress state further inside the criterion; whereas the paths with $d u<0$ allow the stress state to remain along the criterion (even if stresses evolve).

This flow rule determination also allows commenting on the horizontal stress thresholds (for the DEM results) of Figure 5. As evocated in paragraph 3.1, our relations did not recover these stress plateaus. It should now be clear that such stress plateaus should occur when the loading parameters correspond to the material flow rule. However, certain findings suggest that this did not occur for the DEM results of Figure 5. First, the final stress states for these CND discrete simulations do not correspond to the plastic limit criterion deduced from other discrete results (such as the CNL tests), as the maximum ratio $\tau / \sigma$ is in fact lower for these CND discrete simulations than for the CNL discrete simulations [2]. Second, the discrete CND stress levels would correspond to a discrete flow rule with dilatancy angle equal to zero whatever $\sigma$ (see Figures 4 and 5 , in which the levels are reached for different stress states). However, this result contradicts two other sets of discrete results (section 6.4) that reveal a non-null dilatancy angle changing with respect to $\sigma$. Hence, we interpret these thresholds as a bias induced by localisation, once excessive values for $\gamma$ have been reached at the end of certain simulations that use the numerical model.

\section{5 (Non)-Associativity of flow rule}

Figure 22 showed that our two rock joint relations lead to nonassociated behaviour. In the elasto-plastic framework, with direct definitions of the plastic limit criterion $f$ and plastic potential $g$, it is known that nonassociativity cor- 
responds to nonsymmetric constitutive matrices. As our framework is different (no direct definitions of $f$ and $g$, even if such features finally appear), we investigate this possible link between the symmetry of the constitutive matrix and associativity for both the Quadri and INL2 relations.

On the plastic limit criterion, cancellation of the determinants of Quadri matrices is respected for both matrices of the tensorial zones implying $d u<$ 0 (see paragraph 4 and Figure 12). Moreover, for any increment $(d \gamma, d u<$ $0)$, Figure 11 showed that stress response $\overrightarrow{d \sigma}$ to this $(d \gamma, d u<0)$ correspond, either to limit stress state $(\overrightarrow{d \sigma}=\overrightarrow{0}$, when $(d \gamma, d u)$ corresponds to flow rule), or to a neutral loading. Neutral loading means that the rock joint stress state stays during entire loading on the plastic limit criterion (Mohr-Coulomb): $\tau=$ $\sigma \tan (\varphi)$ and $d \tau=d \sigma \tan (\varphi)$. Considering such neutral loadings, and starting from the cancellation of the determinant of corresponding constitutive matrices, equation (9), we obtain:

$$
\begin{aligned}
& \operatorname{det}(M)=0 \Leftrightarrow G_{\gamma}=\frac{G_{u}}{N_{u}} N_{\gamma} \Rightarrow G_{\gamma} d \gamma=\frac{G_{u}}{N_{u}} N_{\gamma} d \gamma \text { by multiplying by } d \gamma \\
& \Leftrightarrow G_{u} d u+G_{\gamma} d \gamma=\frac{G_{u}}{N_{u}} N_{u} d u+\frac{G_{u}}{N_{u}} N_{\gamma} d \gamma \text { by adding } G_{u} d u
\end{aligned}
$$

Expressions for $d \tau$ and $d \sigma$ finally appear (see equation (8), for example), and we find that, on the plastic limit criterion:

$$
d \tau=\frac{G_{u}}{N_{u}} d \sigma \Leftrightarrow \frac{G_{u}}{N_{u}}=\frac{d \tau}{d \sigma}=\tan (\varphi)
$$

Equation (17) is valid for any piecewise linear rock joint relation with constitutive matrices whose determinants vanish along such neutral loadings. Obviously, the chosen expressions for the $G_{u}^{-}$and $N_{u}^{-}$moduli of equation (11) obey equation (17) (as explained previously, the $d u>0$ tensorial zones, then the $G_{u}^{+}$and $N_{u}^{+}$moduli, are not to be considered).

If the matrix is symmetric, we have $N_{\gamma}=G_{u}$, and it appears that the previous equation (17) corresponds to the flow rule definition of equation (14), with $\psi=\varphi$. Symmetry of the constitutive matrix is here also equivalent to an associated behaviour.

To verify this result numerically, for the INL2 relation as well, new expressions for some of moduli are proposed in equation (18). All other expressions remain as in equations (10) and (11). In this case, the symmetry of the $M^{+-}$ matrix of Table 1, which corresponds to $d \gamma>0$ and $d u<0$, is obtained (with the exception of the contractant behaviour), along with of its determinant on 
plasticity limit criterion.

$$
\begin{aligned}
& G_{\gamma}^{+}=\left(G_{\gamma}^{0}-\left(G_{\gamma}^{0}-G_{\gamma}^{f}\right) \frac{1-e^{-\frac{\tau / \sigma}{\tan (\varphi)}}}{1-e^{-1}}\right)\left(\frac{\sigma}{\sigma_{0}}\right)^{\frac{1}{3}} \text { where } G_{\gamma}^{f}=N_{d} N_{u}^{f} \tan (\varphi)^{2} \\
& G_{u}^{-}=N_{d} N_{u}^{f} \tan (\varphi)\left(\frac{\sigma}{\sigma_{0}}\right)^{\frac{1}{3}} \\
& N_{\gamma}^{+}=G_{u}^{-} \text {if } \tau / \sigma>\tan \left(\varphi_{c}\right)
\end{aligned}
$$

Different simulations are then performed using these new "symmetric" Quadri and INL2 relations to determine if an associated behaviour is obtained numerically in these cases.

First, two CNL shearing paths, for $\sigma=1$ and $20 \mathrm{MPa}$, are simulated with both these relations, see Figure 24. Slopes of $u(\gamma)$ curves can be observed in

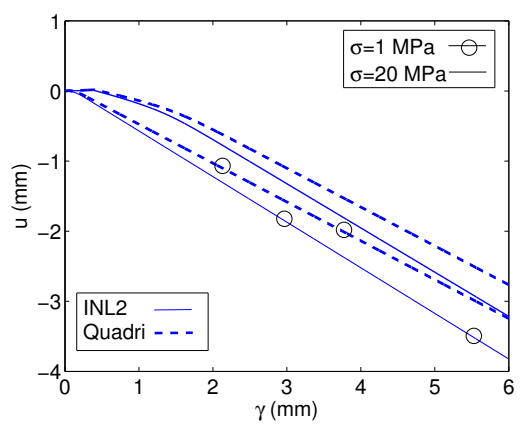

(a) $u(\gamma)$

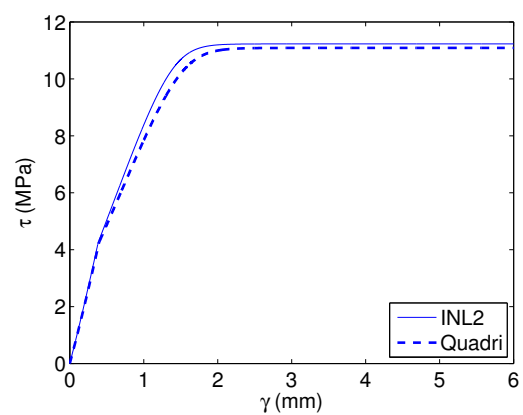

(b) $\tau(\gamma)$ for $\sigma=20 \mathrm{MPa}$

Figure 24: Results of CNL shearing paths $(\sigma=1$ or $20 \mathrm{MPa})$ for symmetric cases of both relations

Figure 24(a). Once the $\tau$-plateau is reached (which occurs in all cases for $\gamma \geq$ $2.5 \mathrm{~mm}$, see Figure 24(b)), the final slopes do not depend anymore on the $\sigma$ values - the curves are parallel - and $\psi=\varphi=29^{\circ}$ is obtained precisely for the Quadri case, and approximately (with slight differences) for the INL2 case. As experiments show that the dilatancy rate of rock joints decreases with $\sigma$ $[37,6,27]$, such results illustrate the need to consider nonassociativity in order when simulating their behaviour, as it has been previously discussed by Plesha [8] for example.

Second, proportional loading paths are used with two different imposed volume variation rates: $R=\tan \left(25^{\circ}\right)<\tan (\varphi)$, and $R=\tan \left(35^{\circ}\right)>\tan (\varphi)$ (Figure 25). Consistent with the previous discussion in paragraph 6.3 and with equality $\psi=\varphi$, an "infinite" increase in stress values is observed for 


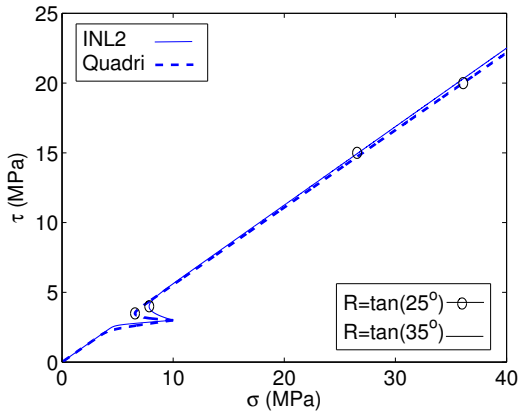

(a) Mohr plane

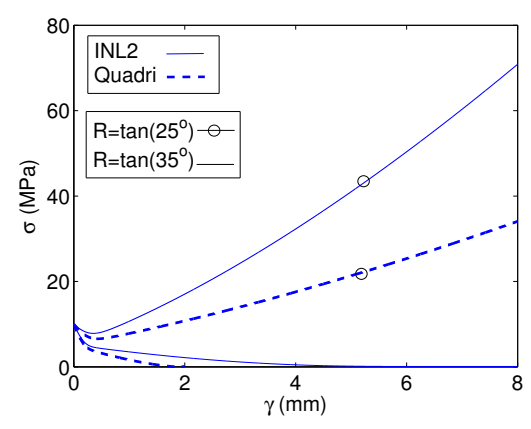

(b) $\sigma(\gamma)$

Figure 25: Proportional displacement loading paths simulated with both relations, from $(\tau=3 \mathrm{MPa} ; \sigma=10 \mathrm{MPa})$. For imposed volume variation rates $R$ around $\tan (\psi)(\psi=\varphi)$

$R=\tan \left(25^{\circ}\right)>\tan (\varphi)$, whereas the stresses end to vanish for $R=\tan \left(35^{\circ}\right)<$ $\tan (\varphi)$

\subsection{Singularity or regularity of the plastic potential}

A more general discussion on the flow rule question can be found in [35], including studies with soils using the INL2 and Octo relations. One issue raised by the corresponding authors, Darve \& Nicot, was the singularity of the flow rule (the plastic potential, strictly). As the constitutive relations used present different tensorial zones, different constitutive matrices exist for a given stress state, depending on the loading direction. For this stress state, it would thus be possible to obtain different plastic strain directions corresponding to different flow rules, depending on the different matrices. This situation would lead to a singular plastic potential with vertex effects, also called "corner theories in elasto-plasticity". Such cases occur for general 3D conditions. While it has been shown that, for rate-independent (i.e., elasto-plastic) materials under 2D conditions (axisymmetry or plane stress for example) a regular flow rule exists at failure as the eigenvector related to the first vanishing eigenvalue of the elastoplastic matrix, when the plastic limit condition is reached with a vanishing value for the determinant of the elasto-plastic matrix. We note that such eigenvectors corresponding to the flow rule were presented in Figure 13, for our case.

Indeed, as the framework has been stated as 2D in this paper, it has been shown that a regular flow rule can be exhibited. As we shown, the loading direction $\overrightarrow{d l}$ leading to $\overrightarrow{d \sigma}=\overrightarrow{0}$ can finally belong to only one tensorial zone. And it is remarkable that in all of the previous discussions, this regular nonassociated flow rule was obtained both by two constitutive relations and by direct discrete simulations. 


\section{Conclusion}

Constitutive modelling of rock joints has been discussed. To obtain powerful constitutive relations, relations with a high number of tensorial zones have been used. A quadrilinear relation (Quadri, with four tensorial zones), and an incrementally non-linear, second order (INL2, with infinite tensorial zones) relation were then proposed. These two relations correspond to what was performed for soils $[3,17]$ with an octolinear (with eight tensorial zones), and also an INL2 relation. The INL2 and Quadri relations were compared throughout the paper to illustrate the differences between these two types of relations, which correspond to two different interpolations. From several perspectives, such differences were generally small (with the exception of the case shown in Figure 21). Nevertheless, the INL2 relation seems to be a little more accurate, whereas the Quadri relation offers the possibility to lead analytical developments. Our relations were calibrated using a numerical database from a discrete model of an infilled rock joint [2]. In addition to a high (even infinite, in the case of INL2) number of tensorial zones, the relations have the advantages of linking normal and tangential variables, and of considering the dilatancy or the effect of the compressions on $\tau$ variations, depending on the shearing state of the joint. The predictive abilities of the relations were also discussed.

The relations are defined outside of the classical elasto-plastic formalism. During calibration, however, proper care was taken to ensure that the proposed constitutive relations respect the theoretical requirements. It was analytically proven and numerically verified that both of the constitutive relations fulfill the continuity condition (or the so-called "consistency condition" in classical elastoplastic theory). The relations also reflect the existences of a plasticity limit criterion and a flow rule, without introducing these features directly. Indeed, analytical and numerical analyses were presented and showed that both our relations obey a Mohr-Coulomb plasticity limit criterion (without realising any correction for stress values). For the flow rule, the values of dilatancy angle were determined for the three different models presented here (Quadri or INL2 relations, or DEM), which were based on infilled rock joints. All the results reveal similar values for the dilatancy angles that decrease according to $\sigma$. It is important to note that our models correspond to nonassociated behaviours. We analytically and numerically illustrated that associativity is obtained for symmetric constitutive matrices, as in classical elasto-plasticity. Associativity should be avoided in rock joint simulations, due to the observed decreasing dilatancy with $\sigma$ for example.

Due to this nonassociativity, different kinds of rock joint failures may occur: especially on or before the plasticity limit criterion [38, 39, 40]. A material stability analysis will thus be led, by use of the "second order work criterion" $[25,41,42]$, which is the only one available for predicting all kinds of failures if flutter instabilities are excluded. Using these two tools, the INL2 constitutive relation (which has been implemented in UDEC [43]), and the second order work criterion, should allow a comprehensive mechanical analysis of rock slope stability. 


\section{References}

[1] Bandis SC, Lumsden AC, Barton NR. Fundamentals of rock joint deformation. Int. J. Rock Mech. Min. Sci. \& Geomech. Abstr. 1983; 20(6):249-268.

[2] Duriez J, Darve F, Donzé FV. A discrete modeling-based constitutive relation for infilled rock joints. International Journal of Rock Mechanics 8 Mining Sciences 2011; 48(3):458-468, doi:10.1016/j.ijrmms.2010.09.008.

[3] Darve F, Labanieh S. Incremental constitutive law for sands and clays. simulations of monotonic and cyclic tests. International Journal for Numerical and Analytical Methods in Geomechanics 1982; 6:243-275.

[4] Darve F. The expression of rheological laws in incremental form and the main classes of constitutive equations. Geomaterials Constitutive Equations and Modelling, Darve F (ed.). Elsevier Applied Science: London, 1990; 123148.

[5] Barton N, Bandis S, Bakhtar K. Strength, deformation and conductivity coupling of rock joints. Int. J. Rock Mech. Min. Sci. 83 Geomech. Abstr. 1985; 22:121-140.

[6] Leichnitz W. Mechanical properties of rock joints. International Journal of Rock Mechanics and Mining Sciences 85 Geomechanics Abstracts 1985; 22(5):313 - 321, doi:10.1016/0148-9062(85)92063-7.

[7] Saeb S, Amadei B. Modelling rock joints under shear and normal loading. Int. J. Rock Mech. Min. Sci. \& Geomech. Abstr. 1992; 29(3):267-278.

[8] Plesha ME. Constitutive models for rock discontinuities with dilatancy and surface degradation. International Journal for Numerical and Analytical Methods in Geomechanics 1987; 11:345-362.

[9] A Gens IC, Alonso EE. A constitutive model for rock joints formulation and numerical implementation. Computers and Geotechnics 1990; 9:3-20.

[10] Wang J, Ichikawab Y, Leung C. A constitutive model for rock interfaces and joints. Int. J. Rock Mech. E Min. Sci. 2003; 40:41-53.

[11] Souley M, Homand F, Amadei B. An extension to the saeb and amadei constitutive model for rock joints to include cyclic loading paths. Int. J. Rock Mech. Min. Sci. \& Geomech. Abstr. 1995; 32:101-109.

[12] Hill R. Continuum micro-mechanics of elastoplastic polycristals. J. of the Mech. and Phys. of Solids 1965; 13:89-101.

[13] Hill R. Generalized relations for incremental deformation of metal crystals by multislip. J. of the Mech. and Phys. of Solids 1966; 14(2):95-102.

[14] Hill R. The essential structure of constitutive laws for metal composites and polycrystals. J. of the Mech. and Phys. of Solids 1967; 15(2):79-95. 
[15] Saada AS, Bianchini G. Constitutive equations for granular non-cohesive soils. International Workshop on Constitutive Equations for Granular Noncohesive Soils (1987: Cleveland, Ohio), AA Balkema, 1989.

[16] Nicot F, Darve F. Basic features of plastic strains: From micro-mechanics to incrementally nonlinear models. International Journal of Plasticity 2007; 23:1555-1588.

[17] Darve F, Flavigny E, Meghachou M. Yield surfaces and principle of superposition: Revisit through incrementally non-linear constitutive relations. International Journal of Plasticity 1995; 11(8):927-948, doi:10.1016/ S0749-6419(95)00037-2.

[18] Darve F, Nicot F. On incremental non-linearity in granular media : phenomenological and multi-scale views (part i). International Journal for $\mathrm{Nu}$ merical and Analytical Methods in Geomechanics 2005; 29:1387-1409.

[19] Nicot F, Lambert C, Darve F. A new constitutive relation for rock joints calibrated by means of a discrete element method. Deformation Characteristics of Geomaterials, Benedetto D, Doanh, Sauzéat (eds.), Swets and Zeitlinger, Lisse, 2003; 1257-1262.

[20] Royis P, Doanh T. Theoretical analysis of strain response envelopes using incrementally non-linear constitutive equations. International Journal for Numerical and Analytical Methods in Geomechanics 1998; 22:97-132.

[21] Cundall P, Strack O. A discrete numerical model for granular assemblies. Géotechnique 1979; 29:47-65.

[22] Kutter HK, Raunterberg A. The residual shear strength of filled joints in rock. Proc. 4th Symp. ISRM, 1979; 221-227.

[23] Papaliangas T, Hencher SR, Lumsden AC, Manolopoulou S. The effect of frictional fill thickness on the shear strength of rock discontinuities. Int. J. Rock Mech. Min. Sci. \&3 Geomech. Abstr. 1993; 30:81-91.

[24] Pereira JP. Rolling friction and shear behaviour of rock discontinuities filled with sand. Int. J. Rock Mech. ES Min. Sci. 1997; 34:244e1-244e17.

[25] Darve F, Servant G, Laouafa F, Khoa H. Failure in geomaterials : continuous and discrete analyses. Comput. Methods Appl. Mech. Engrg. 2004; 193:3057-3085.

[26] Stephansson O, Jing L. Testing and modeling of rock joints. Proc. of the MJFR-2 Conf., Rossmanith (ed.), Balkema, 1995.

[27] Boulon M, Armand G, Hoteit N, Divoux P. Experimental investigations and modelling of shearing of calcite healed discontinuities of granodiorite under typical stresses. Engineering Geology 2002; 64:117-133. 
[28] Smilauer V, Catalano E, Chareyre B, Dorofeenko S, Duriez J, Gladky A, Kozicki J, Modenese C, Scholtès L, Sibille L, et al.. Yade Documentation. 1st edn., The Yade Project, 2010. http://yade-dem.org.

[29] Radjai F, Wolf DE, Jean M, Moreau JJ. Bimodal character of stress transmission in granular packings. Physical Review Letters 1998; 80(1):61-64.

[30] Cambou B, Jean M. Micromécanique des milieux granulaires. Hermes Science, 2001.

[31] Calvetti F, Viggiani G, Tamagnini C. A numerical investigation of the incremental behavior of granular soils. Rivista Italiana di Geotecnica 2003; 3:11-29.

[32] Sibille L, Donzé FV, Nicot F, Chareyre B, Darve F. From bifurcation to failure in a granular material: a dem analysis. Acta Geotechnica 2008; 3(1):15-24.

[33] Donzé FV, Richefeu V, Magnier SA. Advances in discrete element method applied to soil, rock and concrete mechanics. Electronic Journal of Geotechnical Engineering 2009; Special Volume:1-44.

[34] Gudehus G. A comparison of some constitutive laws for soils under radially symmetric loading and unloading. Proc. 3rd Numer. Meth. in Geomechanics, 1979 .

[35] Darve F, Nicot F. On flow rule in granular media : phenomenological and multi-scale views (part ii). International Journal for Numerical and Analytical Methods in Geomechanics 2005; 29:1411-1432.

[36] Darve F. Liquefaction phenomenon of granular materials and constitutive instability. Int. J. of Eng. Comput. 2005; 13:5-28.

[37] Ladanyi B, Archambault G. Simulation of shear behavior of a jointed rock mass. Proc. 11th Symp. on Rock Mech., vol. 1, 1970; 105-125.

[38] Desrues J, Chambon R. Shear band analysis and shear moduli calibration. International Journal of Solids and Structures 2002; 39(13-14):3757-3776.

[39] Darve F, Sibille L, Daouadji A, Nicot F. Bifurcations in granular media: macro- and micro-mechanics approaches. C. R. Mécanique 2007; 335:496515.

[40] Khoa H, Georgopoulos I, Darve F, Laouafa F. Diffuse failure in geomaterials: Experiments and modelling. Computers and Geotechnics 2006; 33:114 .

[41] Sibille L, Nicot F, Donzé FV, Darve F. Material instability in granular assemblies from fundamentally different models. International Journal for Numerical and Analytical Methods in Geomechanics 2007; 31:457-481. 
[42] Nicot F, Darve F. Diffuse and localized failure modes: Two competing mechanisms. International Journal for Numerical and Analytical Methods in Geomechanics 2011; 35(5):586-601, doi:10.1002/nag.912.

[43] Itasca. UDEC Version 4.0: Use Manual 2007. 